Supporting Information for

\title{
Performance evaluation of the three-layer ONIOM method: Case study for a zwitterionic peptide
}

\author{
Keiji Morokuma, ${ }^{1,1}$ Qingfang Wang ${ }^{1}$ and Thom Vreven ${ }^{2}$ \\ ${ }^{1}$ Cherry L. Emerson Center for Scientific Computation and Department of Chemistry, \\ Emory University, Atlanta, GA 30322 \\ ${ }^{2}$ Gaussian, Inc., 340 Quinnipiac Street, Building 40, Wallingford, CT 06492
}

\section{Total of 13 pages}

\section{$X Y Z$ coordinates (in A) for the optimized geometries.}

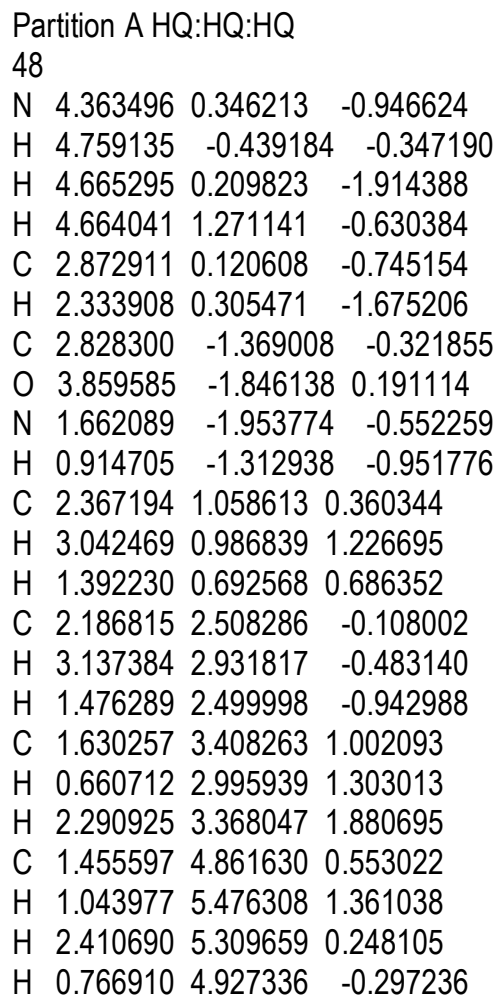

$\begin{array}{llll}\text { C } & 1.156129 & -3.148491 & 0.133350 \\ \text { H } & 1.894171 & -3.517809 & 0.844934 \\ \text { H } & 0.922605 & -3.932542 & -0.597546 \\ \text { C } & -0.099032 & -2.641425 & 0.882266 \\ \text { O } & -0.096480 & -2.484326 & 2.092870 \\ \text { N } & -1.118341 & -2.251774 & 0.042908 \\ \text { H } & -0.906195 & -2.356853 & -0.944862 \\ \text { C } & -1.782303 & -0.949947 & 0.268029 \\ \text { H } & -1.819799 & -0.794970 & 1.348043 \\ \text { C } & -0.904513 & 0.188261 & -0.364568 \\ \text { O } & -0.134170 & -0.166721 & -1.333718 \\ \text { O } & -1.010483 & 1.326344 & 0.116755 \\ \text { C }-3.207935 & -0.966639 & -0.309843 \\ \text { C } & -4.045980 & 0.260921 & 0.070837 \\ \text { C }-5.440656 & 0.253630 & -0.567555 \\ \text { C } & -6.287254 & 1.467345 & -0.170219 \\ \text { H } & -3.705027 & -1.881157 & 0.041060 \\ \text { H } & -3.145135 & -1.044817 & -1.406052 \\ \text { H }-4.147126 & 0.299095 & 1.165486 \\ \text { H }-3.507010 & 1.172017 & -0.212235 \\ \text { H }-5.338351 & 0.222761 & -1.662083 \\ \text { H }-5.969676 & -0.668852 & -0.286364 \\ \text { H }-7.276359 & 1.440698 & -0.642736 \\ \text { H }-6.437273 & 1.506042 & 0.915884 \\ \text { H }-5.798987 & 2.403360 & -0.467945 \\ \text { Partition A HQ:HQ:LQ } & \\ 48 & & \\ \text { N } & 4.599450 & 0.105622 & -0.478231\end{array}$

H $4.783502-0.8367310 .008185$

H $5.0729950 .139239 \quad-1.382038$

H 4.9125320 .8952380 .092052

$\begin{array}{llll}\text { C } 3.070657 & 0.029826 & -0.608166\end{array}$

H $2.7851160 .254183 \quad-1.638359$

C $2.798947 \quad-1.468244 \quad-0.229989$

$\begin{array}{llll}0 & 3.747176 & -2.095602 & 0.287748\end{array}$

N $1.587366 \quad-1.898534 \quad-0.517324$

H $0.894249 \quad-1.172240 \quad-0.889925$

C 2.3852110 .9829730 .323667

H 2.6286610 .7543731 .394751

H 1.2662300 .8297270 .193866

C 2.6850402 .4362430 .015689

H 3.7168902 .7112250 .360440

H $2.6391742 .609417 \quad-1.092135$

C 1.6677353 .3312400 .697440

H 0.6309363 .0323940 .368014

H 1.7191363 .1738171 .806907

C 1.9133784 .7816480 .375858

H 1.1565535 .4201930 .892755

H 2.9306795 .0996600 .709872

H $1.8314124 .961871 \quad-0.723417$

$\begin{array}{llll}\text { C } & 0.984079 & -3.128534 & 0.011144\end{array}$

H $1.644545 \quad-3.5889790 .745389$

H $0.795532 \quad-3.834145 \quad-0.807700$

$\begin{array}{llll}C & -0.321161 & -2.656997 & 0.693618\end{array}$

$\begin{array}{llll}\mathrm{O} & -0.423977 & -2.620699 & 1.910285\end{array}$

N -1.245740 $-2.165093 \quad-0.192363$

H $-0.941220 \quad-2.162197 \quad-1.160557$

\footnotetext{
${ }^{1}$ Corresponding author:orokuma@emory.edu
} 
C -1.922545 -0.8736110 .110394$ H $-1.993998 \quad-0.8026751 .200087$ C - $-1.010010 \quad 0.313087 \quad-0.413706$ $\begin{array}{llll}0 & -0.101963 & -0.020597 & -1.270316\end{array}$ O -1.2161131 .4397550 .042842$ $\begin{array}{llll}\text { C } & -3.309775 & -0.790687 & -0.485140\end{array}$ C $-4.1139780 .310043 \quad 0.177472$ $\begin{array}{llll}\text { C } & -5.457286 & 0.483492 & -0.498529\end{array}$ C -6.2751751 .5579260 .170494$ H $-3.850081 \quad-1.767034 \quad-0.358301$ $\begin{array}{lll}\mathrm{H}-3.236922 & -0.592371 & -1.587498\end{array}$ H -4.2679600 .0627541 .260752$ H -3.5344311 .2723200 .134857$ H $-5.3007920 .747636 \quad-1.577893$

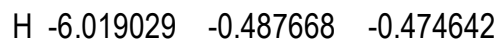
H -7.257351 $1.678908 \quad-0.346770$ H -6.468724 1.2976821 .239256 H -5.7368442 .5361640 .141522$

\section{Partition A HQ:LQ:LQ}

48

N $4.6438810 .149482-0.121650$ H $4.754482 \quad-0.7871360 .375977$ H $5.2896150 .212267 \quad-0.908815$ H $4.8257050 .927790 \quad 0.517330$

$\begin{array}{llll}\text { C } 3.171443 & 0.070017 & -0.552279\end{array}$

H $3.1133510 .269896 \quad-1.625841$

C $2.816852 \quad-1.414023 \quad-0.210382$

$\begin{array}{lllll}0 & 3.651415 & -2.057464 & 0.444702\end{array}$

N $1.657532 \quad-1.864243 \quad-0.703546$

H $1.009652 \quad-1.190813-1.158310$

C 2.3198371 .0413340 .206127

H 2.3565960 .8415641 .309925

H $1.2340090 .884160 \quad-0.115248$

$\begin{array}{lll}\text { C } 2.656668 & 2.490393 & -0.075964\end{array}$

H 3.6809782 .7510970 .300918

H $2.6481212 .672614 \quad-1.183175$

C 1.6298923 .3885170 .588646

H 0.5930793 .0292170 .322697

H 1.7270623 .3000261 .702722

C 1.8077524 .8243550 .172627

H 1.0563125 .4672890 .691957

H $2.8290395 .194093 \quad 0.433311$

H $1.6633054 .937639-0.929188$

$\begin{array}{llll}\text { C } & 1.033614 & -3.084043 & -0.208829\end{array}$

H $1.711096-3.6034830 .524997$

H $0.848075 \quad-3.764913 \quad-1.085224$

C $-0.300530-2.7460380 .499835$

$\begin{array}{llll}\mathrm{C} & -0.400799 & -2.963137 & 1.716951\end{array}$

N $-1.338278 \quad-2.248447 \quad-0.290789$

H $-1.112828 \quad-2.209209-1.268477$

$\begin{array}{llll}\text { C }-1.953451 & -0.989589 & 0.134223\end{array}$

H -2.054391 -1.0115581 .258173$

$\begin{array}{lll}\text { C }-1.052115 & 0.217879 & -0.253003\end{array}$

$\begin{array}{llll}0 & -0.310229 & 0.123063 & -1.294181\end{array}$

$\begin{array}{lllll}0 & -1.018139 & 1.233361 & 0.485031\end{array}$
C $-3.340488 \quad-0.801708 \quad-0.489772$

$\begin{array}{llll}C & -4.092502 & 0.295781 & 0.233558\end{array}$

C $-5.390076 \quad 0.624438 \quad-0.473274$

C $-6.160307 \quad 1.693100 \quad 0.258552$

H $-3.926333 \quad-1.756216 \quad-0.432676$

H -3.225420 $-0.536563 \quad-1.573406$

H $-4.309081 \quad-0.0278651 .285641$

H -3.444282 1.2124550 .296262

H $-5.1690610 .969273 \quad-1.518057$

H $-6.017712 \quad-0.301985-0.557391$

H -7.107932 $1.930235 \quad-0.282414$

H -6.4183801 .3553951 .291408$

H -5.554533 2.6281200 .338401

\section{Partition A LQ:LQ:LQ}

48

N $3.7819450 .345830 \quad-1.548511$

H $4.5949880 .063857 \quad-0.989215$

H $3.767253 \quad-0.185371 \quad-2.419679$

H $3.835773 \quad 1.347053 \quad-1.752876$

$\begin{array}{lll}\text { C } 2.530398 & 0.071554 & -0.766933\end{array}$

H $1.6190430 .240389 \quad-1.453173$

$\begin{array}{llll}\text { C } & 2.599235 & -1.384745 & -0.231834\end{array}$

$\begin{array}{lllll}0 & 3.579282 & -1.705232 & 0.469147\end{array}$

N $1.615893 \quad-2.252807 \quad-0.651160$

H $0.931159-1.850481 \quad-1.281710$

C 2.3832721 .0100770 .428724

H 3.1439790 .7713771 .215791

H 1.3512360 .8002270 .851857

C 2.4474952 .4710090 .036372

H $3.5145002 .804276 \quad-0.066331$

H $1.9346212 .625543 \quad-0.950925$

C 1.7456623 .3247251 .074538

H 0.6591443 .0273751 .101704

H 2.1801213 .1164202 .087170

C 1.8715064 .7910820 .755618

H 1.3407575 .3974721 .529020

H 2.9428295 .1065030 .735259

H $1.4180805 .018227 \quad-0.239598$

$\begin{array}{llll}C & 1.068518 & -3.234381 & 0.255462\end{array}$

H $1.819352 \quad-3.4930191 .053708$

H $0.826256 \quad-4.161279-0.335206$

C $-0.214452 \quad-2.6995490 .940107$

$\begin{array}{llll}0 & -0.252524 & -2.627506 & 2.177394\end{array}$

N $-1.288114 \quad-2.3437990 .115120$

H -1.146864 $-2.572493 \quad-0.851112$

$\begin{array}{llll}C & -1.804592 & -0.981986 & 0.245233\end{array}$

H $-1.841805 \quad-0.7286121 .344880$

$\begin{array}{llll}\text { C } & -0.865932 & 0.039591 & -0.457988\end{array}$

$\begin{array}{llll}0 & -0.272720 & -0.304109 & -1.539244\end{array}$

O -0.6599231 .1585280 .078441$

$\begin{array}{lll}\text { C }-3.212648 & -0.852169 & -0.346219\end{array}$

C -3.853014 0.4405790 .114189

C -5.167866 $0.681014 \quad-0.596283$

$\begin{array}{llll}C & -5.827780 & 1.949134 & -0.119556\end{array}$

H $-3.849718 \quad-1.718726 \quad-0.029065$
H $-3.145561 \quad-0.868843 \quad-1.465652$

H -4.0261670 .4002811 .221904$

H $-3.1486311 .295046 \quad-0.079197$

H $-4.9887430 .743232 \quad-1.702340$

H $-5.853927 \quad-0.189669-0.421637$

H $-6.7902702 .114867 \quad-0.660883$

H -6.0433131 .8937250 .974949$

H $-5.163887 \quad 2.828997 \quad-0.300260$

Partition A HQ:HQ:MM(RESP)

48

N $4.3460660 .052683 \quad-1.114540$

H $4.682477 \quad-0.829217 \quad-0.589796$

H $4.548088 \quad-0.043956 \quad-2.112321$

H $4.7934950 .903578 \quad-0.769106$

$\begin{array}{llll}\text { C } & 2.868253 & -0.037798 & -0.813798\end{array}$

H $2.2498280 .235900 \quad-1.669199$

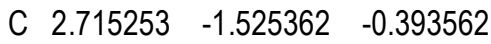

$\begin{array}{llll}0 & 3.757214 & -2.089356 & 0.007718\end{array}$

N $1.491166 \quad-1.994789 \quad-0.507542$

H $0.752173 \quad-1.275771 \quad-0.858670$

C 2.5027440 .9029700 .346732

H 3.2168680 .7774251 .162605

H 1.5123550 .6429230 .724174

$\begin{array}{lll}\text { C } 2.488728 & 2.368927 & -0.108908\end{array}$

H $3.4858592 .663895 \quad-0.437880$

H $1.7927532 .474425 \quad-0.943189$

C 2.0411083 .2898241 .034657

H 1.0462272 .9962191 .371855

H 2.7376343 .1965931 .869022

C 1.9995674 .7515120 .581643

H 1.6801475 .3794551 .413742

H 2.9903935 .0686190 .254948

H $1.2920324 .864345 \quad-0.240685$

$\begin{array}{llll}C & 0.942537 & -3.131605 & 0.238578\end{array}$

H $1.680797 \quad-3.5318790 .933688$

H $0.616582-3.920803-0.450276$

$\begin{array}{llll}C & -0.230271 & -2.479545 & 1.007054\end{array}$

$\begin{array}{llll}0 & -0.126803 & -2.172777 & 2.183922\end{array}$

$\begin{array}{llll}\mathrm{N} & -1.281651 & -2.127574 & 0.195427\end{array}$

$\begin{array}{lll}\mathrm{H}-1.142782 & -2.357046 & -0.782953\end{array}$

$\begin{array}{llll}\text { C } & -1.885631 & -0.788972 & 0.333864\end{array}$

H $-1.987370 \quad-0.5748551 .398096$

$\begin{array}{llll}C & -0.967823 & 0.308041 & -0.321972\end{array}$

$\begin{array}{llll}0 & -0.135454 & -0.125475 & -1.216039\end{array}$

O -1.0929211 .4663370 .067163$

$\begin{array}{lll}\text { C }-3.293630 & -0.831469 & -0.289606\end{array}$

$\begin{array}{llll}C & -4.107726 & 0.444952 & -0.027872\end{array}$

$\begin{array}{llll}C & -5.544276 & 0.293684 & -0.547285\end{array}$

C $-6.362551 \quad 1.563514 \quad-0.296283$

H $-3.834289-1.6771830 .139130$

$\begin{array}{llll}\text { H } & -3.206332 & -0.989211 & -1.365933\end{array}$

H -4.133074 0.6415821 .045083

H $-3.639298 \quad 1.288857 \quad-0.534425$

$\begin{array}{llll}H & -5.523551 & 0.092563 & -1.619325\end{array}$

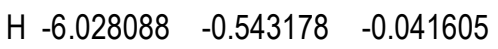


H $-7.3750591 .423366 \quad-0.675527$

H -6.4078081 .7704770 .773500$

H $-5.9031152 .408763 \quad-0.809923$

Partition A HQ:HQ:MM(RESP) (EE) 48

N $4.3469070 .043712 \quad-1.116128$

H $4.677090-0.843422 \quad-0.593238$

H $4.548940 \quad-0.050555 \quad-2.114075$

H $4.7985040 .890965 \quad-0.767383$

$\begin{array}{llll}\text { C } & 2.868504 & -0.040949 & -0.815508\end{array}$

H $2.2510130 .235078 \quad-1.670775$

C $2.710543 \quad-1.528246 \quad-0.396187$

$\begin{array}{lllll}0 & 3.752787 & -2.096040 & 0.001597\end{array}$

N $1.484971 \quad-1.992666 \quad-0.505935$

H $0.743965 \quad-1.268339 \quad-0.854406$

C $2.506460 \quad 0.9007720 .345304$

H 3.2203550 .7725921 .160969

H 1.5153230 .6440000 .723003

$\begin{array}{llll}\text { C } 2.497183 & 2.366860 & -0.109824\end{array}$

H $3.4953822 .658863 \quad-0.438223$

H $1.8018292 .474896 \quad-0.944298$

C $2.0518433 .288307 \quad 1.034157$

H 1.0557352 .9977021 .370363

H 2.7474473 .1920531 .868948

C 2.0155004 .7504590 .582320

H 1.6975215 .3788471 .414653

H $3.0076125 .064640 \quad 0.256662$

H $1.3090244 .866411-0.240496$

$\begin{array}{llll}\text { C } & 0.937297 & -3.131033 & 0.238294\end{array}$

H $1.675745-3.5326210 .932603$

H $0.611340 \quad-3.919239 \quad-0.451850$

C $-0.235704 \quad-2.4802971 .007881$

$\begin{array}{llll}\mathrm{C} & -0.132063 & -2.175292 & 2.185372\end{array}$

N -1.285999 -2.1259460 .196634$

H $-1.147308 \quad-2.356678 \quad-0.781514$

$\begin{array}{llll}\text { C }-1.888991 & -0.786668 & 0.336134\end{array}$

H $-1.992272 \quad-0.574517 \quad 1.400582$

C $\begin{array}{llll}-0.968998 & 0.309720 & -0.315799\end{array}$

$\begin{array}{llll}0 & -0.133996 & -0.123362 & -1.209222\end{array}$

O -1.0950591 .4678980 .071614$

$\begin{array}{llll}\text { C } & -3.295457 & -0.826443 & -0.290061\end{array}$

$\begin{array}{lll}\text { C }-4.109362 & 0.449823 & -0.027535\end{array}$

$\begin{array}{lll}\text { C }-5.544273 & 0.299542 & -0.551439\end{array}$

C $-6.3633051 .568795 \quad-0.300339$

H $-3.837619-1.6726810 .135746$

H -3.206040 $-0.982099 \quad-1.366523$

H -4.137637 0.6440541 .045784

H -3.639405 $1.294666-0.531078$

H $-5.5201950 .100484 \quad-1.623785$

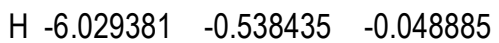

H -7.374699 $1.429377 \quad-0.682834$

H -6.4117731 .7736590 .769713$

H $-5.9024532 .415120 \quad-0.810939$

Partition A HQ:LQ:MM(RESP)
48

N $4.1796020 .254220 \quad-1.301821$

H $4.704712 \quad-0.439843 \quad-0.714017$

H $4.3195850 .020780 \quad-2.288071$

H $4.5212281 .204135 \quad-1.141471$

$\begin{array}{llll}\text { C } & 2.749690 & 0.031157 & -0.860488\end{array}$

H $2.0342200 .268157 \quad-1.661913$

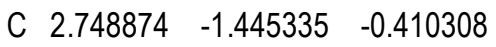

$\begin{array}{lllll}0 & 3.795766 & -1.878164 & 0.083181\end{array}$

$\begin{array}{llll}\text { N } & 1.595674 & -2.099544 & -0.641370\end{array}$

H $0.852427 \quad-1.544846 \quad-1.109958$

C 2.4393890 .9424540 .341634

H 3.2339300 .8523421 .084742

H 1.5094550 .6186910 .810161

C $2.3028122 .411800 \quad-0.085166$

H $3.2411162 .762180 \quad-0.516443$

H $1.5156722 .491600 \quad-0.837019$

C 1.9422833 .2974901 .115708

H 1.0124922 .9464471 .564532

H 2.7365793 .2377951 .861036

C 1.7671124 .7577100 .690819

H 1.5144355 .3608051 .563242

H 2.6932575 .1322640 .253832

H $0.961961 \quad 4.836147 \quad-0.040733$

$\begin{array}{llll}\text { C } & 1.072542 & -3.134435 & 0.237052\end{array}$

H $1.843400 \quad-3.4466330 .993369$

H $0.786698 \quad-4.023497 \quad-0.389219$

$\begin{array}{llll}C & -0.168789 & -2.568752 & 0.976255\end{array}$

$\begin{array}{llll}0 & -0.137761 & -2.472709 & 2.211845\end{array}$

N -1.269841 -2.2074310 .194496$

H -1.155012 $-2.428658 \quad-0.776966$

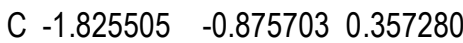

H $-1.949292 \quad-0.6730201 .457493$

C $-0.933501 \quad 0.240604 \quad-0.239891$

$\begin{array}{llll}0 & -0.169248 & -0.019904 & -1.237092\end{array}$

$\begin{array}{lllll}0 & -0.945045 & 1.371883 & 0.298382\end{array}$

$\begin{array}{llll}\text { C } & -3.238133 & -0.878287 & -0.310211\end{array}$

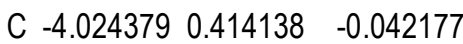

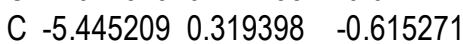

$\begin{array}{llll}C & -6.234997 & 1.605898 & -0.358079\end{array}$

H $-3.809113 \quad-1.7181260 .089843$

H -3.128417 $-1.017318 \quad-1.387144$

H -4.0817610 .5824981 .034307$

H $-3.5132511 .257175 \quad-0.507757$

H $-5.3920370 .146381 \quad-1.691086$

H $-5.970439-0.515959-0.150076$

H $-7.2369271 .506040 \quad-0.776174$

H -6.312431 1.7856650 .714766

H $-5.7333582 .450416 \quad-0.831895$

\section{Partition A HQ:MM:MM(RESP)}

48

$\begin{array}{llll}\text { N } & 4.363902 & -0.013020 & -1.218981\end{array}$

H $4.772797 \quad-0.859899 \quad-0.740960$

H $4.517986 \quad-0.097502 \quad-2.228925$

H $4.8194660 .845385 \quad-0.893899$

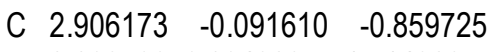

H $2.2937930 .204828 \quad-1.714928$

C $2.706427 \quad-1.579004 \quad-0.451030$

$\begin{array}{llll}0 & 3.697834 & -2.220657 & -0.120823\end{array}$

$\begin{array}{llll}\text { N } & 1.436517 & -1.998731 & -0.511924\end{array}$

H $\quad 0.717467 \quad-1.322189 \quad-0.763462$

C 2.5912400 .8530710 .313380

H 3.3105370 .6912921 .118225

H 1.5962670 .6272550 .699913

$\begin{array}{lll}\text { C } 2.627385 & 2.324291 & -0.125623\end{array}$

H $3.6273012 .582561 \quad-0.476121$

H $1.9159462 .468621 \quad-0.940776$

C 2.2489073 .2502671 .038801

H 1.2507922 .9973671 .397828

H 2.9608733 .1165391 .854419

C 2.2615714 .7177380 .602891

H 1.9902155 .3490671 .449382

H 3.2574624 .9948080 .255823

H $1.5404094 .871686 \quad-0.200690$

$\begin{array}{llll}\text { C } & 0.871276 & -3.112547 & 0.248197\end{array}$

H $1.570322 \quad-3.4493011 .014248$

H $0.620646 \quad-3.938933 \quad-0.415017$

$\begin{array}{llll}C & -0.395845 & -2.589788 & 0.919524\end{array}$

$\begin{array}{llll}0 & -0.476571 & -2.532593 & 2.140533\end{array}$

N $-1.327497 \quad-2.0954460 .101508$

H $-1.150789 \quad-2.219340 \quad-0.882110$

$\begin{array}{llll}\text { C } & -1.974487 & -0.803945 & 0.375332\end{array}$

H - $-2.174401 \quad-0.7141621 .444448$

$\begin{array}{llll}C & -1.038274 & 0.357062 & -0.040927\end{array}$

$\begin{array}{llll}0 & -0.362193 & 0.218137 & -1.090913\end{array}$

$\begin{array}{lllll}0 & -0.953055 & 1.342076 & 0.720958\end{array}$

$\begin{array}{lll}\text { C }-3.309360 & -0.741366 & -0.383473\end{array}$

$\begin{array}{llll}C & -4.115871 & 0.522180 & -0.046023\end{array}$

C $-5.4773090 .507534 \quad-0.754762$

$\begin{array}{llll}C & -6.287779 & 1.764891 & -0.427409\end{array}$

H $-3.903659-1.615840 \quad-0.112969$

H $-3.117898 \quad-0.772387 \quad-1.457520$

H -4.2731510 .5701501 .032652$

H $-3.5619651 .406353 \quad-0.363554$

$\begin{array}{llll}H & -5.324570 & 0.456486 & -1.833747\end{array}$

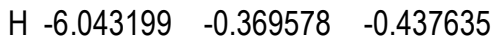

H $-7.2465451 .723264 \quad-0.944792$

H $-6.464564 \quad 1.8232460 .647144$

H $-5.7444432 .652242 \quad-0.754145$

Partition A HQ:MM:MM(RESP) (EE) 48

$\begin{array}{llll}\text { N } & 4.491889 & -0.028614 & -1.032082\end{array}$

$\begin{array}{llll}\text { H } & 4.828822 & -0.881302 & -0.508939\end{array}$

H $4.768684 \quad-0.116071 \quad-2.014945$

H $4.9158340 .823104 \quad-0.652391$

$\begin{array}{llll}\text { C } 2.997112 & -0.091109 & -0.847112\end{array}$

H $2.4849640 .201732 \quad-1.765534$

$\begin{array}{lll}\text { C } 2.742427 & -1.568756 & -0.451390\end{array}$

$\begin{array}{llll}0 & 3.690440 & -2.217705 & -0.016104\end{array}$

$\begin{array}{llll}\text { N } & 1.478589 & -1.974517 & -0.637337\end{array}$ 
H $0.793547 \quad-1.273606 \quad-0.947105$

$\begin{array}{llll}\text { C } 2.554944 & 0.863998 & 0.275129\end{array}$

H 3.1838840 .7115141 .154074

H 1.5257730 .6392630 .556780

C $2.6381492 .330595 \quad-0.172208$

H $3.6677812 .582107 \quad-0.429056$

H $2.0074122 .469486 \quad-1.052081$

C 2.1563923 .2682790 .943610

H 1.1278333 .0229611 .209445

H 2.7878353 .1387651 .823657

C 2.2176184 .7316850 .498571

H 1.8710975 .3716591 .310514

H 3.2431445 .0018180 .244786

H $1.5761214 .882315-0.370511$

C $0.850208 \quad-3.0484950 .132500$

H $1.568657-3.5109830 .809603$

H $0.438433 \quad-3.800943 \quad-0.537834$

C $-0.274501-2.4166300 .954403$

$\begin{array}{llll}0 & -0.050171 & -2.044399 & 2.101147\end{array}$

N -1.436399 $-2.169596 \quad 0.336549$

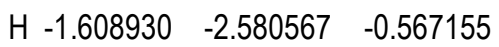

C -2.039674 -0.8301890 .463898$

H $-2.186518 \quad-0.601907 \quad 1.521381$

C $-1.0958830 .246118 \quad-0.132100$

$\begin{array}{llll}0 & -0.402738 & -0.071595 & -1.132877\end{array}$

O $-\begin{array}{llll}0.979898 & 1.331142 & 0.468858\end{array}$

$\begin{array}{lll}\text { C }-3.410382 & -0.826751 & -0.231060\end{array}$

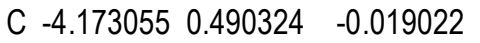

C $-5.5689820 .422337 \quad-0.653612$

C $-6.3352641 .732504 \quad-0.451524$

H $-4.008112 \quad-1.6440690 .176054$

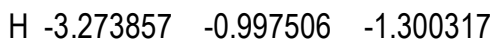

H -4.273624 0.6785451 .051014

H $-3.6183201 .311973 \quad-0.473407$

H $-5.4723230 .230400 \quad-1.723153$

H $-6.135440 \quad-0.392006 \quad-0.199730$

$\begin{array}{llll}H & -7.319990 & 1.651454 & -0.912438\end{array}$

H $-6.455636 \quad 1.931747 \quad 0.613907$

H $-5.7913382 .556205 \quad-0.915223$

\footnotetext{
Partition A LQ:LQ:MM(RESP)

48

N $3.418973 \quad-0.040257 \quad-1.896911$

H $4.308883 \quad-0.443999-1.585960$

H $3.096138 \quad-0.539006 \quad-2.730978$

H $3.5499620 .947409 \quad-2.127748$

$\begin{array}{lll}\text { C } 2.400512 & -0.169000 & -0.824708\end{array}$

H $1.3742700 .164638 \quad-1.243354$

$\begin{array}{llll}\text { C } 2.365550 & -1.634147 & -0.328676\end{array}$

$\begin{array}{lllll}0 & 3.358458 & -2.072384 & 0.281803\end{array}$

$\begin{array}{llll}\text { N } & 1.270536 & -2.393341 & -0.683747\end{array}$

H $0.589226 \quad-1.918825 \quad-1.266548$

C 2.7088700 .7851070 .379016

H 3.6816520 .5385330 .807567

H 1.9449820 .6287601 .144780

C $2.7070752 .264359-0.040132$
}

H $3.5478012 .459111-0.706902$

H $1.7788712 .489227 \quad-0.567431$

C 2.8193523 .1788501 .187269

H 1.9769692 .9957201 .855795

H 3.7463262 .9602641 .719204

C 2.8159174 .6541470 .778177

H 2.8954945 .2772901 .669295

H 3.6620194 .8605270 .121973

H 1.8868424 .8926960 .259097

C $0.680937 \quad-3.3062550 .267646$

H $1.454493 \quad-3.6572311 .006648$

$\begin{array}{llll}\text { H } & 0.283507 & -4.194572 & -0.297551\end{array}$

C $-0.468476 \quad-2.6154771 .043661$

$\begin{array}{llll}\mathrm{O} & -0.374328 & -2.486672 & 2.272398\end{array}$

N -1.581006 $-2.176763 \quad 0.307918$

H $-1.548369 \quad-2.464542 \quad-0.653457$

C $-1.917086 \quad-0.7653590 .401573$

H $-1.911301 \quad-0.4640381 .485886$

C $-0.9021360 .110320-0.361165$

$\begin{array}{llll}0 & -0.468632 & -0.288301 & -1.502591\end{array}$

$\begin{array}{llll}0 & -0.4486666 & 1.154638 & 0.168632\end{array}$

$\begin{array}{llll}\text { C } & -3.359788 & -0.576012 & -0.162333\end{array}$

C -3.868262 0.8608600 .028288

C $-5.3145561 .000345 \quad-0.466356$

C $-5.828568 \quad 2.431126 \quad-0.283026$

H $-4.032606 \quad-1.2569260 .361863$

H $-3.370048 \quad-0.826378-1.224568$

H -3.824165 1.1188941 .087515

H $-3.234873 \quad 1.550423 \quad-0.531027$

$\begin{array}{llll}H & -5.363074 & 0.740281 & -1.524710\end{array}$

H $-5.957708 \quad 0.319702 \quad 0.093231$

H $-6.8556452 .496299-0.642912$

H $-5.8027802 .702367 \quad 0.772944$

H -5.207076 $3.124465 \quad-0.850800$

Partition A LQ:MM:MM(RESP)

48

N $4.0577480 .231706 \quad-1.541788$

H $4.801608 \quad-0.217135 \quad-0.981603$

H $4.077893 \quad-0.151795 \quad-2.491753$

H $4.214852 \quad 1.243377 \quad-1.580200$

$\begin{array}{lll}\text { C } 2.743669 & -0.051701 & -0.911695\end{array}$

H $1.9355470 .167454 \quad-1.669586$

C $2.699757 \quad-1.525495 \quad-0.419386$

$\begin{array}{lllll}0 & 3.728645 & -2.000619 & 0.092413\end{array}$

N $1.534278 \quad-2.207844 \quad-0.622319$

H $0.787308 \quad-1.698529 \quad-1.058379$

C 2.5050390 .8809470 .325796

H 3.3341180 .7752601 .027599

H 1.5884110 .5696780 .832120

$\begin{array}{llll}\text { C } & 2.369494 & 2.353923 & -0.089033\end{array}$

H $3.2998712 .700395 \quad-0.539989$

H $1.5648032 .444435 \quad-0.820756$

C 2.0453443 .2343561 .126038

H 1.1238622 .8876321 .595035

H 2.8570273 .1634761 .851386
C 1.8713754 .6986390 .714813

H 1.6447295 .2979171 .596963

H $2.7895145 .068950 \quad 0.257783$

H 1.0492414 .7880320 .003679

C $1.011469 \quad-3.1333800 .363339$

H $1.778940-3.4079371 .087818$

H $0.625136 \quad-4.028223 \quad-0.121973$

C $-0.121181-2.407051 \quad 1.081383$

$\begin{array}{llll}0 & 0.018794 & -2.020178 & 2.235576\end{array}$

N $-1.187414 \quad-2.110336 \quad 0.336443$

H $-1.186002 \quad-2.478698 \quad-0.600067$

C $-1.835765 \quad-0.7935290 .423866$

H $-1.948604 \quad-0.5134641 .472551$

C $-0.965904 \quad 0.282362 \quad-0.271069$

$\begin{array}{llll}0 & -0.340147 & -0.046903 & -1.309280\end{array}$

$\begin{array}{llll}0 & -0.884327 & 1.405080 & 0.268243\end{array}$

$\begin{array}{llll}\text { C } & -3.230508 & -0.880635 & -0.215818\end{array}$

C $-4.039627 \quad 0.413952 \quad-0.041392$

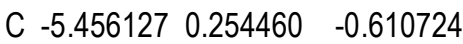

$\begin{array}{llll}C & -6.269684 & 1.541191 & -0.445207\end{array}$

H $-3.777896 \quad-1.6983140 .256256$

H -3.128001 $-1.102467 \quad-1.279519$

H -4.1046950 .6563041 .020487$

H $-3.5401451 .231531 \quad-0.562298$

H $-5.3955170 .008644 \quad-1.671869$

H $-5.968238 \quad-0.556224 \quad-0.090489$

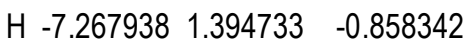

H -6.354770 1.7930820 .612442

H $-5.7811552 .359916 \quad-0.974718$

Partition A MM:MM:MM(RESP)

48

N $4.0881340 .129679 \quad-1.507373$

H $4.815957 \quad-0.134604 \quad-0.856636$

$\begin{array}{llll}\text { H } & 4.142702 & -0.476959 & -2.312140\end{array}$

H $4.190273 \quad 1.095394 \quad-1.781894$

$\begin{array}{lll}\text { C } 2.788430 & -0.069041 & -0.839712\end{array}$

H $1.9806100 .130703 \quad-1.546433$

$\begin{array}{llll}\text { C } & 2.669279 & -1.517253 & -0.382942\end{array}$

$\begin{array}{llll}0 & 3.641497 & -2.086996 & 0.105199\end{array}$

$\begin{array}{llll}\text { N } & 1.471725 & -2.072830 & -0.546099\end{array}$

H $0.773281 \quad-1.490222 \quad-0.994151$

C 2.6112970 .8759370 .361085

H 3.4584270 .7682601 .040794

H 1.7030570 .5963280 .900157

C $2.4893412 .341397 \quad-0.083563$

H $3.4105902 .659219 \quad-0.573031$

H $1.6618682 .430548 \quad-0.789845$

C 2.2205973 .2555361 .119976

H 1.3047272 .9408241 .621519

H 3.0506193 .1812161 .823915

C 2.0689524 .7143050 .681158

H 1.8807115 .3376901 .555612

H 2.9823795 .0529310 .191273

H $1.2297894 .807848 \quad-0.009228$

$\begin{array}{llll}\text { C } & 0.917563 & -3.168374 & 0.244053\end{array}$ 
H $1.628816 \quad-3.4977531 .002134$

$\begin{array}{llll}H & 0.645722 & -4.004115 & -0.398597\end{array}$

C $-0.330492 \quad-2.6201540 .931363$

$\begin{array}{llll}0 & -0.381109 & -2.524757 & 2.151642\end{array}$

$\begin{array}{llll}\mathrm{N} & -1.280063 & -2.143957 & 0.122955\end{array}$

$\begin{array}{llll}\mathrm{H}-1.133978 & -2.306016 & -0.859842\end{array}$

C - $-1.891782 \quad-0.8290890 .364670$

H -2.046608 $-0.688598 \quad 1.435827$

$\begin{array}{lll}\text { C }-0.951550 & 0.293144 & -0.139761\end{array}$

$\begin{array}{llll}0 & -0.335265 & 0.102295 & -1.217782\end{array}$

O -0.8036461 .3018380 .580339$

$\begin{array}{llll}\text { C } & -3.254964 & -0.772164 & -0.342369\end{array}$

$\begin{array}{lll}\text { C }-4.021811 & 0.522157 & -0.030114\end{array}$

C $-5.4100540 .507034 \quad-0.684746$

$\begin{array}{llll}\text { C } & -6.181227 & 1.794921 & -0.382098\end{array}$

$\begin{array}{lll}\text { H }-3.854712 & -1.620881 & -0.009161\end{array}$

H $-3.108189 \quad-0.854757 \quad-1.420705$

H -4.135502 0.6200751 .050648

H $-3.4629101 .379459-0.407050$

H $-5.300900 \quad 0.406179 \quad-1.765505$

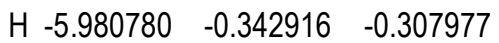

H $-7.1602181 .752184 \quad-0.860002$

H -6.3144331 .9034110 .694841$

H $-5.6331622 .655142 \quad-0.768094$

\section{Partition B HQ:HQ:HQ}

48

N $4.363496 \quad 0.346213 \quad-0.946624$

H $4.759135 \quad-0.439184-0.347190$

H $4.6652950 .209823 \quad-1.914388$

H $4.6640411 .271141 \quad-0.630384$

C $2.8729110 .120608 \quad-0.745154$

H $2.3339080 .305471 \quad-1.675206$

$\begin{array}{llll}\text { C } & 2.828300 & -1.369008 & -0.321855\end{array}$

$\begin{array}{llll}0 & 3.859585 & -1.846138 & 0.191114\end{array}$

N $1.662089-1.953774 \quad-0.552259$

H $0.914705 \quad-1.312938-0.951776$

C $2.3671941 .058613 \quad 0.360344$

H 3.0424690 .9868391 .226695

H 1.3922300 .6925680 .686352

$\begin{array}{llll}\text { C } 2.186815 & 2.508286 & -0.108002\end{array}$

H $3.1373842 .931817-0.483140$

H $1.4762892 .499998-0.942988$

C 1.6302573 .4082631 .002093

H 0.6607122 .9959391 .303013

H 2.2909253 .3680471 .880695

C $1.455597 \quad 4.861630 \quad 0.553022$

H 1.0439775 .4763081 .361038

H 2.4106905 .3096590 .248105

H $0.7669104 .927336 \quad-0.297236$

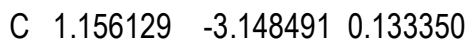

H $1.894171 \quad-3.5178090 .844934$

$\begin{array}{llll}\text { H } & 0.922605 & -3.932542 & -0.597546\end{array}$

C $-0.099032 \quad-2.6414250 .882266$

$\begin{array}{llll}0 & -0.096480 & -2.484326 & 2.092870\end{array}$

N -1.118341 -2.2517740 .042908$
H $-0.906195 \quad-2.356853 \quad-0.944862$

$\begin{array}{llll}\text { C }-1.782303 & -0.949947 & 0.268029\end{array}$

H $-1.819799 \quad-0.7949701 .348043$

$\begin{array}{llll}\text { C }-0.904513 & 0.188261 & -0.364568\end{array}$

$\begin{array}{llll}0 & -0.134170 & -0.166721 & -1.333718\end{array}$

O -1.0104831 .3263440 .116755$

$\begin{array}{lll}\text { C }-3.207935 & -0.966639 & -0.309843\end{array}$

C -4.0459800 .2609210 .070837$

C $-5.4406560 .253630 \quad-0.567555$

C $-6.2872541 .467345 \quad-0.170219$

H $-3.705027-1.8811570 .041060$

H $-3.145135 \quad-1.044817 \quad-1.406052$

H -4.147126 0.2990951 .165486

H $-3.5070101 .172017-0.212235$

H $-5.3383510 .222761 \quad-1.662083$

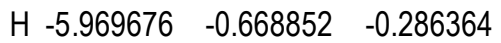

H -7.276359 $1.440698 \quad-0.642736$

H -6.4372731 .5060420 .915884$

H $-5.7989872 .403360 \quad-0.467945$

Partition B HQ:HQ:LQ

48

N $4.5701320 .105137 \quad-0.394383$

H $4.713402 \quad-0.7841940 .186839$

H $\begin{array}{llll}5.144390 & 0.056580 & -1.236794\end{array}$

H 4.8181090 .9495660 .124126

$\begin{array}{lll}\text { C } 3.075562 & 0.008286 & -0.666072\end{array}$

H $2.8997330 .191754 \quad-1.729312$

$\begin{array}{llll}\text { C } & 2.783494 & -1.464140 & -0.271088\end{array}$

$\begin{array}{lllll}0 & 3.663176 & -2.057959 & 0.387543\end{array}$

N $1.622405 \quad-1.963748 \quad-0.670174$

H $0.959484-1.311686-1.131626$

C 2.3063221 .0164660 .193767

H 2.4525060 .7778051 .254031

H $1.2275750 .857317 \quad-0.011206$

C $2.6132592 .490695 \quad-0.098281$

H 3.6514622 .7482590 .170840

H $2.5171232 .673822 \quad-1.177540$

C 1.6501493 .4148380 .662005

H 0.6164373 .1183370 .409780

H 1.7601003 .2392401 .739835

C 1.8696444 .8965970 .350326

H 1.1619995 .5183520 .905662

H 2.8813175 .2202800 .621706

H $1.7262765 .103299 \quad-0.716382$

$\begin{array}{llll}\text { C } & 1.001966 & -3.158814 & -0.074839\end{array}$

H $1.707208-3.6168580 .612736$

H $0.738160 \quad-3.863046 \quad-0.868710$

C $-0.230516-2.6453150 .703358$

$\begin{array}{llll}0 & -0.209915 & -2.552801 & 1.915450\end{array}$

N -1.287347 $-2.247076 \quad-0.114555$

H $-1.104870 \quad-2.307585-1.117303$

C $-1.930670 \quad-0.9584440 .198464$

H -2.021455 -0.8801611 .320114$

$\begin{array}{llll}\text { C } & -1.053866 & 0.224469 & -0.304791\end{array}$

$\begin{array}{llll}0 & -0.298417 & 0.044093 & -1.324002\end{array}$
O $-1.057867 \quad 1.3093420 .327496$

$\begin{array}{llll}\text { C } & -3.323432 & -0.861471 & -0.429965\end{array}$

C $-4.0979770 .281406 \quad 0.192382$

C $-5.4069460 .511119 \quad-0.532204$

C -6.1993531 .6247660 .102373$

$\begin{array}{lll}\text { H }-3.887084 & -1.819405 & -0.280187\end{array}$

H $-3.220479-0.696328 \quad-1.534536$

H $-4.3007510 .053558 \quad 1.271935$

H -3.471344 1.2147420 .163368

H $-5.1999820 .761953 \quad-1.606248$

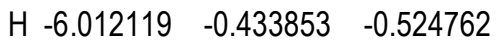

H $-7.1552331 .787649 \quad-0.451371$

H -6.443815 1.3789781 .164098

H -5.6161292 .5772940 .090194$

\section{Partition B HQ:LQ:LQ}

48

$\begin{array}{llll}\mathrm{N} & 4.643880 & 0.149482 & -0.121650\end{array}$

H $4.754482 \quad-0.7871360 .375978$

H $\begin{array}{lll}5.289615 & 0.212267 & -0.908814\end{array}$

H 4.8257050 .9277890 .517331

$\begin{array}{llll}\text { C } 3.171443 & 0.070017 & -0.552279\end{array}$

H $3.1133520 .269895 \quad-1.625841$

C $2.816852 \quad-1.414023 \quad-0.210382$

$\begin{array}{llll}0 & 3.651415 & -2.057464 & 0.444703\end{array}$

N $1.657532 \quad-1.864244 \quad-0.703546$

H $1.009652 \quad-1.190813 \quad-1.158310$

C 2.3198371 .0413340 .206127

H 2.3565950 .8415641 .309924

H $1.2340090 .884160 \quad-0.115249$

$\begin{array}{lll}\text { C } 2.656668 & 2.490393 & -0.075964\end{array}$

H 3.6809782 .7510970 .300918

H $2.6481212 .672614 \quad-1.183175$

C 1.6298923 .3885170 .588646

H 0.5930793 .0292170 .322697

H 1.7270623 .3000261 .702721

C 1.8077534 .8243560 .172627

H 1.0563135 .4672890 .691957

H 2.8290395 .1940930 .433311

H $1.6633064 .937639 \quad-0.929188$

$\begin{array}{llll}\text { C } & 1.033614 & -3.084043 & -0.208829\end{array}$

H $1.711095 \quad-3.6034840 .524997$

H $0.848075 \quad-3.764914 \quad-1.085224$

$\begin{array}{llll}\text { C } & -0.300530 & -2.746039 & 0.499835\end{array}$

$\begin{array}{llll}0 & -0.400799 & -2.963137 & 1.716951\end{array}$

N -1.338278 $-2.248447 \quad-0.290789$

H $-1.112829 \quad-2.209209 \quad-1.268477$

C $-1.953451 \quad-0.9895890 .134222$

H - $-2.054391 \quad-1.0115581 .258173$

$\begin{array}{llll}C & -1.052115 & 0.217879 & -0.253004\end{array}$

$\begin{array}{llll}0 & -0.310229 & 0.123063 & -1.294182\end{array}$

O -1.0181381 .2333610 .485030$

$\begin{array}{lll}\text { C }-3.340488 & -0.801708 & -0.489772\end{array}$

C -4.0925020 .2957820 .233558$

$\begin{array}{llll}\text { C } & -5.390076 & 0.624439 & -0.473274\end{array}$

C -6.1603071 .6931010 .258553$ 
H $-3.926334 \quad-1.756216 \quad-0.432676$

H $-3.225421 \quad-0.536563 \quad-1.573406$

H $-4.309081 \quad-0.027864 \quad 1.285641$

H -3.444282 1.2124550 .296262

H $-5.1690610 .969273 \quad-1.518057$

H $-6.017713 \quad-0.301984 \quad-0.557390$

H -7.107932 $1.930236 \quad-0.282413$

H -6.4183791 .3553961 .291409$

H -5.554532 2.6281210 .338402

\section{Partition B LQ:LQ:LQ}

48

N $3.781944 \quad 0.345829 \quad-1.548511$

H $4.5949870 .063856-0.989216$

H $3.767251-0.185373 \quad-2.419680$

H $3.8357721 .347052-1.752878$

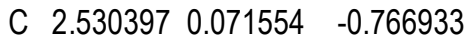

H $1.6190410 .240388 \quad-1.453172$

$\begin{array}{llll}\text { C } & 2.599234 & -1.384745 & -0.231834\end{array}$

$\begin{array}{llll}0 & 3.579281 & -1.705232 & 0.469148\end{array}$

N $1.615892 \quad-2.252807 \quad-0.651159$

H $0.931159 \quad-1.850482 \quad-1.281710$

C 2.3832721 .0100770 .428724

H 3.1439800 .7713761 .215791

H 1.3512360 .8002270 .851858

C 2.4474962 .4710090 .036372

H $3.5145012 .804275 \quad-0.066331$

H $1.9346212 .625543 \quad-0.950925$

C 1.7456633 .3247251 .074538

H 0.6591453 .0273761 .101703

H 2.1801223 .1164202 .087170

C 1.8715084 .7910820 .755618

H 1.3407595 .3974721 .529020

H 2.9428325 .1065030 .735260

H $1.4180835 .018228 \quad-0.239598$

C $1.068518-3.2343820 .255462$

H $1.819352 \quad-3.4930191 .053708$

H $0.826255 \quad-4.161279-0.335206$

$\begin{array}{llll}\text { C }-0.214453 & -2.699549 & 0.940108\end{array}$

$\begin{array}{llll}0 & -0.252525 & -2.627506 & 2.177394\end{array}$

N -1.288114 -2.3437990 .115120$

H $-1.146864 \quad-2.572493 \quad-0.851112$

$\begin{array}{llll}\text { C }-1.804592 & -0.981986 & 0.245232\end{array}$

H $-1.841804 \quad-0.7286111 .344880$

$\begin{array}{llll}\text { C } & -0.865932 & 0.039590 & -0.457990\end{array}$

$\begin{array}{llll}0 & -0.272721 & -0.304110 & -1.539246\end{array}$

$\begin{array}{llll}0 & -0.659921 & 1.158528 & 0.078439\end{array}$

$\begin{array}{llll}\text { C } & -3.212648 & -0.852169 & -0.346219\end{array}$

C -3.853014 0.4405800 .114189

C $-5.1678650 .681015 \quad-0.596282$

$\begin{array}{llll}\text { C }-5.827779 & 1.949136 & -0.119555\end{array}$

H $-3.849718 \quad-1.718725 \quad-0.029064$

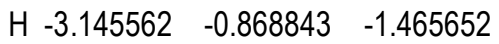

H -4.0261650 .4002831 .221904$

H -3.148630 $1.295046 \quad-0.079198$

H $-4.9887440 .743233 \quad-1.702339$

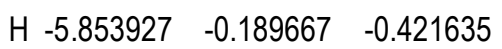

H $-6.7902692 .114868 \quad-0.660881$

H -6.0433101 .8937280 .974950$

H $-5.1638862 .828999 \quad-0.300260$

Partition B HQ:HQ:MM(RESP)

48

N $4.419189-0.175192 \quad-1.100754$

H $4.761995 \quad-0.936583 \quad-0.462032$

H $4.661194 \quad-0.427196 \quad-2.063817$

H $4.8482190 .727870 \quad-0.877183$

$\begin{array}{lll}\text { C } 2.926019 & -0.177822 & -0.854050\end{array}$

H $2.4189040 .063730 \quad-1.793122$

$\begin{array}{lll}\text { C } 2.662919 & -1.629240 & -0.380086\end{array}$

$\begin{array}{lllll}0 & 3.588985 & -2.237117 & 0.159436\end{array}$

N $1.419297 \quad-2.081601 \quad-0.597539$

H $0.783310 \quad-1.417428 \quad-1.034683$

C 2.5810260 .8459340 .241371

H 3.1436110 .5929041 .150120

H 1.5277890 .7001260 .499044

C $2.8059442 .313019 \quad-0.158598$

H $3.8709722 .508540 \quad-0.367938$

H $2.2655232 .524299 \quad-1.092362$

C 2.3434033 .2893390 .934497

H 1.2780433 .1172491 .136349

H 2.8740853 .0638381 .869008

C 2.5668954 .7553330 .551864

H 2.2197585 .4195941 .348736

H 3.6288554 .9673680 .381591

H $2.0199195 .016893 \quad-0.361407$

$\begin{array}{llll}\text { C } & 0.762054 & -3.174060 & 0.140547\end{array}$

H $1.485681 \quad-3.6180220 .821654$

H $0.397974 \quad-3.928708 \quad-0.563930$

C $-0.372525 \quad-2.5034180 .942049$

$\begin{array}{llll}0 & -0.280234 & -2.294463 & 2.132396\end{array}$

N -1.448376 -2.1093670 .154052$

$\begin{array}{lll}\mathrm{H}-1.333837 & -2.202871 & -0.854252\end{array}$

$\begin{array}{lll}\text { C }-2.039689 & -0.778359 & 0.378975\end{array}$

H $-2.170835 \quad-0.6157691 .450045$

C $-1.0956210 .322288 \quad-0.165122$

$\begin{array}{lllll}0 & -0.442159 & 0.070481 & -1.208530\end{array}$

$\begin{array}{lllll}0 & -0.985627 & 1.377715 & 0.492648\end{array}$

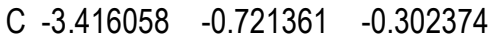

C $-4.1694780 .582753 \quad 0.001815$

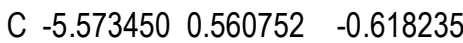

$\begin{array}{llll}C & -6.331681 & 1.858063 & -0.323188\end{array}$

$\begin{array}{llll}\mathrm{H} & -4.015193 & -1.560112 & 0.056232\end{array}$

H $-3.290435 \quad-0.823195 \quad-1.381730$

H $-4.2563130 .703376 \quad 1.082772$

H $-3.616677 \quad 1.429343 \quad-0.406755$

H $-5.4912860 .437383 \quad-1.699047$

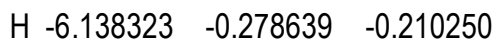

H $-7.3222601 .810140 \quad-0.776061$

H -6.437979 1.9889990 .754247

H $-5.7896972 .707725 \quad-0.739934$
Partition B HQ:HQ:MM(RESP) (EE)

48

N $4.418916 \quad-0.173089 \quad-1.100509$

H $4.761817 \quad-0.934835 \quad-0.462134$

$\begin{array}{llll}\text { H } & 4.661203 & -0.424342 & -2.063688\end{array}$

H $4.847457 \quad 0.730029 \quad-0.876256$

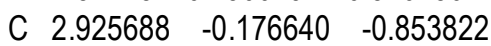

H $2.4183890 .064796 \quad-1.792813$

$\begin{array}{lll}\text { C } 2.663519 & -1.628293 & -0.380112\end{array}$

$\begin{array}{llll}0 & 3.590182 & -2.235656 & 0.159146\end{array}$

N $1.420187 \quad-2.081280 \quad-0.597234$

H $0.783659 \quad-1.417397 \quad-1.034047$

C 2.5802980 .8467010 .241830

H 3.1443160 .5946111 .149968

H 1.5276330 .6993320 .500805

C $2.8025162 .314025 \quad-0.158695$

H $3.8669482 .510952 \quad-0.369925$

H $2.2602102 .524343 \quad-1.091569$

C 2.3403333 .2898520 .934973

H 1.2755803 .1162681 .138583

H 2.8728993 .0653241 .868661

C 2.5609744 .7560920 .551659

H 2.2141795 .4199871 .348987

H 3.6223334 .9696990 .379465

H $2.011967 \quad 5.016625 \quad-0.360677$

C $0.763579 \quad-3.1740410 .141049$

H $1.487416 \quad-3.6172990 .822395$

H $0.400188 \quad-3.929102 \quad-0.563345$

$\begin{array}{llll}C & -0.371606 & -2.503700 & 0.942102\end{array}$

$\begin{array}{llll}0 & -0.279447 & -2.294281 & 2.132422\end{array}$

$\mathrm{N}-1.447165 \quad-2.1101300 .153863$

H $-1.332870 \quad-2.204836 \quad-0.854340$

$\begin{array}{llll}C & -2.039085 & -0.779434 & 0.378372\end{array}$

H $-2.170761 \quad-0.6168601 .449382$

$\begin{array}{llll}C & -1.095150 & 0.321559 & -0.165314\end{array}$

$\begin{array}{llll}0 & -0.441579 & 0.070256 & -1.208771\end{array}$

O $-\begin{array}{llll}0.985372 & 1.376779 & 0.492829\end{array}$

$\begin{array}{lll}\text { C }-3.415088 & -0.723069 & -0.303548\end{array}$

C $-4.168216 \quad 0.580917 \quad 0.001247$

C $-5.572358 \quad 0.559600 \quad-0.618420$

$\begin{array}{llll}C & -6.329375 & 1.857151 & -0.322350\end{array}$

H $-4.014167 \quad-1.5619960 .054741$

H -3.289127 $-0.824447 \quad-1.382907$

H -4.2544400 .7009361 .082295$

H $-3.6150861 .427381 \quad-0.407051$

H $-5.4899570 .436460 \quad-1.699187$

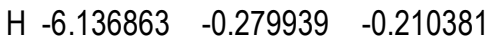

H $-7.320233 \quad 1.810143 \quad-0.774802$

H -6.435412 1.9877450 .755164

H $-5.7872992 .706816 \quad-0.738997$

Partition B HQ:LQ:MM(RESP)

48

N $4.677121 \quad-0.197425 \quad-0.687457$

$\begin{array}{llll}\text { H } & 4.865907 & -1.052439 & -0.102722\end{array}$

H $5.065064 \quad-0.340076 \quad-1.623861$ 
H $\begin{array}{lll}5.109956 & 0.634562 & -0.272534\end{array}$

$\begin{array}{llll}\text { C } 3.152108 & -0.127589 & -0.704168\end{array}$

H $2.8288870 .128281 \quad-1.718007$

$\begin{array}{llll}\text { C } 2.738569 & -1.577337 & -0.290665\end{array}$

$\begin{array}{lllll}0 & 3.577594 & -2.280901 & 0.262466\end{array}$

$\begin{array}{llll}\mathrm{N} & 1.479794 & -1.924067 & -0.632831\end{array}$

H $0.886379-1.209855 \quad-1.054015$

$\begin{array}{llll}\text { C } 2.639811 & 0.892112 & 0.276893\end{array}$

H 3.0278360 .6887271 .313246

H 1.5232620 .7880230 .334437

C $2.9751342 .314239-0.129955$

H $4.0749592 .506619 \quad-0.022766$

H $2.7054292 .479808 \quad-1.206756$

C 2.2167643 .3056450 .731965

H 1.1137363 .1617540 .585876

H 2.4386563 .1087991 .814348

C 2.5907284 .7240570 .385610

H 2.0111715 .4362901 .022492

H 3.6791584 .9029370 .559507

H $2.3622124 .944230-0.685236$

$\begin{array}{llll}\text { C } & 0.786293 & -3.078165 & -0.078399\end{array}$

H $1.457619-3.6154170 .650776$

$\begin{array}{llll}H & 0.524310 & -3.777218 & -0.922031\end{array}$

$\begin{array}{llll}\text { C } & -0.490936 & -2.625561 & 0.660678\end{array}$

$\begin{array}{llll}0 & -0.576703 & -2.775482 & 1.886237\end{array}$

N $-1.522954 \quad-2.103134-0.116942$

H -1.295063 $-1.993667 \quad-1.089410$

C $-2.111515 \quad-0.8308930 .286031$

H $-2.262709 \quad-0.8224891 .366825$

C -1.145262 $0.320720 \quad-0.080584$

$\begin{array}{llll}0 & -0.487170 & 0.208927 & -1.145337\end{array}$

O -1.0162691 .2605500 .730996$

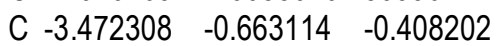

C -4.2188230 .5945510 .062630$

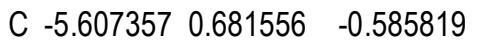

C $-6.3581551 .934307 \quad-0.125446$

$\begin{array}{lll}\text { H }-4.086768 & -1.536629 & -0.183432\end{array}$

$\begin{array}{llll}H & -3.324783 & -0.612924 & -1.488460\end{array}$

H -4.330683 $0.560508 \quad 1.147478$

H $-3.6468281 .482900 \quad-0.207515$

H $-5.5001590 .712678 \quad-1.671023$

H $-6.191287 \quad-0.199241 \quad-0.315029$

H -7.337824 $1.965856 \quad-0.602827$

H $-6.489326 \quad 1.9116260 .956953$

H $-5.7967582 .826824 \quad-0.404012$

Partition B HQ:MM:MM(RESP)
$\begin{array}{llll}48 & & & \\ \text { N } & 4.363902 & -0.013020 & -1.218981 \\ \text { H } & 4.772797 & -0.859899 & -0.740960 \\ \text { H } & 4.517986 & -0.097502 & -2.228925 \\ \text { H } & 4.819466 & 0.845385 & -0.893899 \\ \text { C } & 2.906173 & -0.091610 & -0.859725 \\ \text { H } & 2.293793 & 0.204828 & -1.714928 \\ \text { C } & 2.706427 & -1.579004 & -0.451030 \\ \text { O } & 3.697834 & -2.220657 & -0.120823\end{array}$
N $1.436517 \quad-1.998731 \quad-0.511924$

H $0.717467 \quad-1.322189 \quad-0.763462$

C 2.5912400 .8530710 .313380

H 3.3105370 .6912921 .118225

H 1.5962670 .6272550 .699913

$\begin{array}{llll}\text { C } & 2.627385 & 2.324291 & -0.125623\end{array}$

H $3.6273012 .582561 \quad-0.476121$

H $1.9159462 .468621 \quad-0.940776$

C 2.2489073 .2502671 .038801

H 1.2507922 .9973671 .397828

H 2.9608733 .1165391 .854419

C 2.2615714 .7177380 .602891

H 1.9902155 .3490671 .449382

H 3.2574634 .9948080 .255823

H $1.5404094 .871686 \quad-0.200690$

$\begin{array}{llll}\text { C } & 0.871276 & -3.112547 & 0.248197\end{array}$

H $1.570322 \quad-3.4493011 .014248$

$\begin{array}{llll}\mathrm{H} & 0.620646 & -3.938933 & -0.415017\end{array}$

C $-0.395845 \quad-2.5897880 .919524$

$\begin{array}{llll}0 & -0.476571 & -2.532593 & 2.140533\end{array}$

N $-1.327497 \quad-2.095446 \quad 0.101508$

H -1.150789 $-2.219340-0.882110$

$\begin{array}{llll}\text { C }-1.974487 & -0.803945 & 0.375332\end{array}$

H - $2.174401 \quad-0.7141621 .444448$

$\begin{array}{llll}C & -1.038274 & 0.357062 & -0.040927\end{array}$

$\begin{array}{llll}0 & -0.362193 & 0.218137 & -1.090913\end{array}$

$\begin{array}{llll}0 & -0.953055 & 1.342076 & 0.720958\end{array}$

$\begin{array}{lll}\text { C }-3.309360 & -0.741366 & -0.383473\end{array}$

$\begin{array}{llll}C & -4.115871 & 0.522180 & -0.046023\end{array}$

C $-5.4773090 .507534 \quad-0.754762$

C $-6.2877791 .764891 \quad-0.427409$

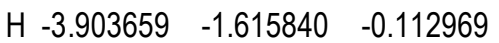

H $-3.117898 \quad-0.772387 \quad-1.457520$

H -4.2731510 .5701501 .032652$

H $-3.5619651 .406353 \quad-0.363554$

$\begin{array}{llll}H & -5.324570 & 0.456486 & -1.833747\end{array}$

H $-6.043199 \quad-0.369578 \quad-0.437635$

H $-7.2465451 .723264 \quad-0.944792$

H $-6.4645641 .823246 \quad 0.647144$

H $-5.7444432 .652242 \quad-0.754145$

Partition B HQ:MM:MM(RESP) (EE) 48

N $4.491889 \quad-0.028614 \quad-1.032083$

H $4.828822 \quad-0.881301 \quad-0.508939$

H $4.7686844-0.116071 \quad-2.014945$

H $4.9158330 .823104 \quad-0.652391$

$\begin{array}{lll}\text { C } 2.997111 & -0.091109 & -0.847113\end{array}$

H $2.484963 \quad 0.201733 \quad-1.765534$

$\begin{array}{llll}\text { C } & 2.742427 & -1.568756 & -0.451389\end{array}$

$\begin{array}{llll}0 & 3.690440 & -2.217705 & -0.016104\end{array}$

$\begin{array}{llll}\text { N } & 1.478589 & -1.974517 & -0.637337\end{array}$

H $0.793547 \quad-1.273606 \quad-0.947106$

C 2.5549430 .8639980 .275129

H 3.1838840 .7115141 .154074

H 1.5257730 .6392630 .556781
C $2.6381492 .330595 \quad-0.172208$

H $3.6677812 .582107 \quad-0.429057$

H $2.0074122 .469486 \quad-1.052081$

C 2.1563923 .2682790 .943610

H 1.1278333 .0229611 .209446

H 2.7878353 .1387651 .823657

C 2.2176184 .7316850 .498571

H 1.8710975 .3716591 .310514

H 3.2431445 .0018180 .244786

H $1.576120 \quad 4.882315 \quad-0.370511$

C $0.850208 \quad-3.0484950 .132500$

H $1.568657 \quad-3.5109830 .809604$

$\begin{array}{llll}H & 0.438433 & -3.800943 & -0.537834\end{array}$

C $-0.274501 \quad-2.4166300 .954403$

$\begin{array}{llll}0 & -0.050171 & -2.044399 & 2.101147\end{array}$

N $-1.436399 \quad-2.169596 \quad 0.336549$

H $-1.608930 \quad-2.580567 \quad-0.567155$

C $-2.039674 \quad-0.8301890 .463898$

H - $-2.186518 \quad-0.6019071 .521381$

C $-1.0958830 .246118 \quad-0.132100$

$\begin{array}{llll}0 & -0.402738 & -0.071596 & -1.132877\end{array}$

O -0.9798971 .3311420 .468858$

$\begin{array}{lll}\text { C }-3.410381 & -0.826751 & -0.231060\end{array}$

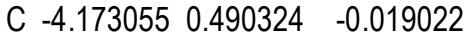

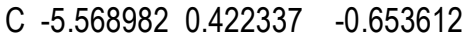

$\begin{array}{llll}C & -6.335264 & 1.732504 & -0.451524\end{array}$

H $-4.008112 \quad-1.6440690 .176054$

$\begin{array}{llll}H & -3.273856 & -0.997506 & -1.300317\end{array}$

H -4.2736240 .6785451 .051014$

H $-3.618320 \quad 1.311973 \quad-0.473407$

H $-5.4723230 .230400 \quad-1.723153$

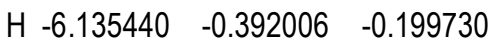

H $-7.3199901 .651454 \quad-0.912438$

H -6.455636 1.9317460 .613907

H $-5.791338 \quad 2.556205 \quad-0.915223$

\section{Partition B LQ:LQ:MM(RESP)}

48

N $4.0845820 .167594 \quad-1.507349$

H $4.818782 \quad-0.219910 \quad-0.896943$

H $4.089359-0.327038 \quad-2.402521$

H $4.2535061 .167389 \quad-1.659701$

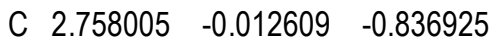

H $1.9636370 .212333 \quad-1.610843$

C $2.665472 \quad-1.469427 \quad-0.292311$

$\begin{array}{llll}0 & 3.610201 & -1.896979 & 0.391129\end{array}$

N $1.571378-2.209460 \quad-0.681123$

H $0.924706 \quad-1.728304 \quad-1.278922$

C 2.5845700 .9369630 .351539

H 3.4137250 .7847781 .095097

H 1.6317140 .6442770 .871409

C $2.5039272 .387371 \quad-0.070925$

H $3.4871072 .739414 \quad-0.480924$

H $1.7354942 .508152 \quad-0.880611$

C 2.1282953 .2643811 .108714

H 1.1561842 .9135661 .545550 
H 2.9070083 .1643611 .910839

C 2.0052604 .7080380 .694297

H 1.7302585 .3351721 .577349

H 2.9702105 .0876350 .280058

H $1.2131824 .830675 \quad-0.083733$

C $0.966711 \quad-3.1846550 .197138$

H $1.716241 \quad-3.5303640 .965781$

H $0.645560 \quad-4.067754 \quad-0.424731$

$\begin{array}{llll}\text { C }-0.251211 & -2.579010 & 0.931327\end{array}$

$\begin{array}{llll}0 & -0.241962 & -2.480608 & 2.165365\end{array}$

N $-1.339397 \quad-2.1926530 .150805$

H $-1.205571 \quad-2.293519 \quad-0.838117$

$\begin{array}{lll}\text { C }-1.894411 & -0.857471 & 0.343700\end{array}$

H $-1.980077 \quad-0.6498691 .411703$

C $-0.9540310 .202163 \quad-0.282992$

$\begin{array}{llll}0 & -0.340748 & -0.106621 & -1.335124\end{array}$

O $-\begin{array}{llll}0.802892 & 1.283722 & 0.321220\end{array}$

$\begin{array}{llll}\text { C } & -3.295829 & -0.802558 & -0.285105\end{array}$

C $-4.0141360 .526613 \quad-0.005410$

$\begin{array}{lll}\text { C }-5.441700 & 0.506311 & -0.569057\end{array}$

$\begin{array}{llll}C & -6.164556 & 1.829007 & -0.298980\end{array}$

H -3.894701 -1.6147080 .130732$

$\begin{array}{llll}H & -3.215254 & -0.949964 & -1.363533\end{array}$

H -4.055956 0.6922531 .072259

H -3.463465 $1.345556 \quad-0.469438$

H $-5.4044990 .337455 \quad-1.646199$

$\begin{array}{llll}H & -6.004707 & -0.304702 & -0.104980\end{array}$

H -7.172998 $1.781744 \quad-0.710626$

H -6.2257612 .0058880 .775394$

H $-5.6245892 .650313 \quad-0.771461$

\section{Partition B LQ:MM:MM(RESP)}

48

N $4.057748 \quad 0.231706 \quad-1.541788$

H $4.801608 \quad-0.217135 \quad-0.981603$

H $4.077894 \quad-0.151794 \quad-2.491752$

H $4.2148531 .243377 \quad-1.580199$

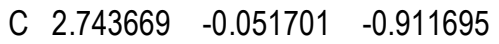

H $1.9355470 .167454 \quad-1.669586$

C $2.699757 \quad-1.525495 \quad-0.419386$

$\begin{array}{llll}0 & 3.728645 & -2.000618 & 0.092413\end{array}$

$\begin{array}{llll}\mathrm{N} & 1.534278 & -2.207844 & -0.622319\end{array}$

H $0.787308 \quad-1.698529 \quad-1.058379$

C 2.5050390 .8809470 .325796

H 3.3341180 .7752601 .027599

H 1.5884110 .5696780 .832120

C $2.3694932 .353923 \quad-0.089033$

H $3.2998712 .700395 \quad-0.539989$

H $1.5648032 .444435 \quad-0.820756$

C 2.0453443 .2343561 .126038

H 1.1238612 .8876321 .595035

H 2.8570273 .1634761 .851386

C 1.8713754 .6986390 .714813

H 1.6447295 .2979171 .596963

H $2.7895135 .068950 \quad 0.257783$

H 1.0492414 .7880320 .003679
$\begin{array}{llll}C & 1.011469 & -3.133380 & 0.363339\end{array}$

H $1.778940-3.4079371 .087818$

H $0.625136 \quad-4.028223 \quad-0.121973$

C $-0.121181 \quad-2.4070511 .081383$

$\begin{array}{lllll}0 & 0.018794 & -2.020178 & 2.235576\end{array}$

$\begin{array}{llll}\mathrm{N} & -1.187414 & -2.110336 & 0.336443\end{array}$

H -1.186001 $-2.478698 \quad-0.600067$

$\begin{array}{llll}C & -1.835765 & -0.793529 & 0.423866\end{array}$

H $-1.948604 \quad-0.5134641 .472550$

C $-0.965904 \quad 0.282362 \quad-0.271069$

$\begin{array}{llll}0 & -0.340147 & -0.046903 & -1.309280\end{array}$

$\begin{array}{lllll}0 & -0.884328 & 1.405080 & 0.268243\end{array}$

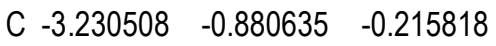

$\begin{array}{llll}C & -4.039627 & 0.413952 & -0.041392\end{array}$

$\begin{array}{llll}C & -5.456128 & 0.254459 & -0.610724\end{array}$

$\begin{array}{llll}C & -6.269684 & 1.541191 & -0.445207\end{array}$

H $-3.777896 \quad-1.6983140 .256256$

H $-3.128001 \quad-1.102467 \quad-1.279519$

H -4.104695 0.6563041 .020487

H $-3.5401451 .231531 \quad-0.562298$

H $-5.395517 \quad 0.008644 \quad-1.671869$

H $-5.968238 \quad-0.556224 \quad-0.090489$

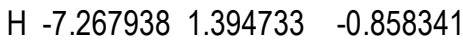

H -6.3547701 .7930810 .612442$

H $-5.7811552 .359916 \quad-0.974718$

Partition B MM:MM:MM(RESP)

48

N $4.088134 \quad 0.129679 \quad-1.507373$

H $4.815957 \quad-0.134604-0.856636$

H $4.142702 \quad-0.476959-2.312140$

H $4.190274 \quad 1.095394 \quad-1.781894$

$\begin{array}{lll}\text { C } 2.788430 & -0.069041 & -0.839712\end{array}$

H $1.9806100 .130703 \quad-1.546433$

$\begin{array}{llll}\text { C } & 2.669279 & -1.517253 & -0.382942\end{array}$

$\begin{array}{lllll}0 & 3.641497 & -2.086996 & 0.105199\end{array}$

N $1.471725 \quad-2.072829 \quad-0.546099$

H $0.773281 \quad-1.490222 \quad-0.994151$

C 2.6112970 .8759370 .361085

H 3.4584270 .7682601 .040794

H 1.7030570 .5963280 .900157

C $2.4893412 .341397 \quad-0.083563$

H $3.4105902 .659219 \quad-0.573031$

H $1.6618682 .430548 \quad-0.789845$

C 2.2205973 .2555361 .119976

H 1.3047272 .9408241 .621518

H 3.0506193 .1812161 .823915

C 2.0689524 .7143050 .681158

H 1.8807115 .3376891 .555612

H 2.9823795 .0529310 .191273

H $1.2297894 .807848 \quad-0.009228$

$\begin{array}{llll}\text { C } & 0.917563 & -3.168374 & 0.244053\end{array}$

H $1.628816 \quad-3.4977531 .002134$

H $0.645722 \quad-4.004115 \quad-0.398597$

C $-0.330492 \quad-2.6201540 .931363$

$\begin{array}{llll}0 & -0.381109 & -2.524757 & 2.151642\end{array}$
N -1.280063 $-2.143957 \quad 0.122955$

H $-1.133978 \quad-2.306016 \quad-0.859842$

$\begin{array}{llll}\text { C }-1.891782 & -0.829089 & 0.364670\end{array}$

H $-2.046608 \quad-0.6885981 .435827$

C $-0.951550 \quad 0.293144 \quad-0.139761$

$\begin{array}{llll}0 & -0.335265 & 0.102295 & -1.217782\end{array}$

O

$\begin{array}{llll}\text { C } & -3.254964 & -0.772164 & -0.342369\end{array}$

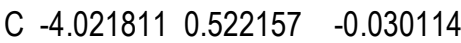

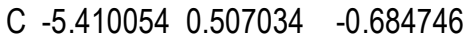

$\begin{array}{llll}C & -6.181227 & 1.794921 & -0.382098\end{array}$

$\begin{array}{lll}\text { H }-3.854712 & -1.620881 & -0.009161\end{array}$

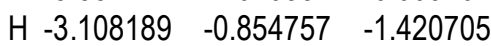

H -4.1355020 .6200751 .050648$

H $-3.4629101 .379459 \quad-0.407050$

H $-5.300900 \quad 0.406179 \quad-1.765506$

H $-5.980780 \quad-0.342916 \quad-0.307977$

H $-7.1602181 .752184 \quad-0.860002$

H -6.3144331 .9034110 .694841$

H $-5.633162 \quad 2.655142 \quad-0.768094$

Partition B HQ:HQ:MM(MULLIKEN)

48

N $4.496939-0.190175 \quad-1.029532$

H $4.796798 \quad-1.001021 \quad-0.428689$

H $4.744410 \quad-0.391884 \quad-2.002350$

H $4.9426650 .686841 \quad-0.746412$

$\begin{array}{llll}\text { C } & 2.998927 & -0.159607 & -0.808916\end{array}$

H $2.4998090 .097069 \quad-1.749605$

$\begin{array}{llll}\text { C } 2.685840 & -1.609248 & -0.363931\end{array}$

$\begin{array}{llll}0 & 3.602191 & -2.276279 & 0.123079\end{array}$

N $1.418713 \quad-2.000942 \quad-0.549959$

H $0.789507 \quad-1.273597 \quad-0.923040$

C 2.6307010 .8680480 .272491

H 3.1202690 .6069671 .217664

H 1.5537650 .7470980 .446139

C $2.8792552 .332648 \quad-0.122249$

H $3.9545332 .555387 \quad-0.201439$

H $2.4404752 .503265-1.116600$

C 2.2287403 .3012660 .879157

H 1.1492623 .0789020 .897657

H 2.6027743 .0900011 .888460

C 2.4531624 .7740370 .525233

H 1.9477105 .4218551 .246568

H 3.5179205 .0301000 .527022

H $2.047927 \quad 5.000657 \quad-0.467822$

C $0.756438 \quad-3.1360250 .119902$

H $1.467822 \quad-3.5929110 .804364$

$\begin{array}{llll}\text { H } & 0.431521 & -3.863187 & -0.630729\end{array}$

$\begin{array}{llll}\text { C } & -0.419312 & -2.523484 & 0.905882\end{array}$

$\begin{array}{llll}0 & -0.384545 & -2.368582 & 2.106856\end{array}$

N $-1.472432 \quad-2.1045650 .095655$

H $-1.298651 \quad-2.136409 \quad-0.909700$

$\begin{array}{llll}\text { C }-2.050749 & -0.777726 & 0.349527\end{array}$

H $-2.177848 \quad-0.6229831 .423775$

C $-1.0724700 .313974 \quad-0.152502$ 
$\begin{array}{llll}0 & -0.359703 & 0.043215 & -1.153485\end{array}$

$\begin{array}{llll}0 & -0.981959 & 1.369668 & 0.506603\end{array}$

C $-3.419870 \quad-0.724078 \quad-0.349730$

C $-4.1921830 .551706 \quad 0.021612$

C $-5.5567900 .561039-0.675533$

C $-6.3146341 .838024 \quad-0.294868$

H $-4.002934-1.596193 \quad-0.045933$

H $-3.271043 \quad-0.762203 \quad-1.430940$

H $-4.3318580 .591448 \quad 1.104562$

H -3.618299 $1.433846 \quad-0.279299$

H $-5.4180360 .525205-1.759572$

H $-6.135223 \quad-0.314561 \quad-0.368603$

H $-7.2877291 .851712 \quad-0.788203$

H -6.4679101 .8776800 .785143$

H $-5.7503752 .719064 \quad-0.606448$

Partition B HQ:HQ:MM(MULLIKEN) (EE) 48

N $4.484424 \quad-0.169693 \quad-1.065072$

H $4.802823 \quad-0.974701-0.468200$

H $4.714619-0.372284 \quad-2.041990$

H $4.9270920 .712292 \quad-0.792827$

C $2.990574-0.149302 \quad-0.819437$

H $2.4730050 .109300 \quad-1.749917$

C $2.690820-1.602091-0.376338$

$\begin{array}{llll}0 & 3.614165 & -2.266523 & 0.098294\end{array}$

N $1.423070 \quad-2.000537 \quad-0.553894$

H $0.787244 \quad-1.272399-0.915600$

C $2.6315980 .871503 \quad 0.271824$

H 3.1299800 .6078641 .211316

H 1.5557880 .7453310 .454439

C $2.8677722 .339627-0.118183$

H $3.9401162 .571528-0.202502$

H $2.4201112 .510489-1.108769$

C 2.2136113 .2986940 .890243

H 1.1346353 .0678080 .907847

H 2.5884073 .0833711 .898147

C 2.4260814 .7754110 .544926

H 1.9145455 .4138031 .270327

H 3.4880745 .0405240 .547690

H $2.0172825 .003315-0.446481$

$\begin{array}{llll}\text { C } & 0.769581 & -3.137771 & 0.120468\end{array}$

H $1.485832-3.5931840 .800459$

H $0.438740 \quad-3.865279 \quad-0.626973$

C $-0.401289-2.5296410 .915483$

O $-0.364100-2.3801842 .116355$

N $-1.462840 \quad-2.1109730 .110185$

H $-1.290979-2.128416 \quad-0.895919$

C $-2.040273 \quad-0.7828180 .370858$

H $-2.161504-0.6289301 .445882$

C $-1.0682240 .311546 \quad-0.135648$

$\begin{array}{llll}0 & -0.359911 & 0.042863 & -1.140254\end{array}$

$\begin{array}{llll}0 & -0.978384 & 1.367755 & 0.522587\end{array}$

C $-3.415263 \quad-0.724697 \quad-0.320496$

C -4.1913150 .5634380 .012212$

C $-5.5576790 .537895 \quad-0.680187$
C $-6.3215411 .822576 \quad-0.340595$

H $-4.001835 \quad-1.5868890 .004069$

H $-3.271100 \quad-0.792000 \quad-1.400873$

H $-4.3341450 .644727 \quad 1.093148$

H $-3.6306641 .443018-0.323529$

H $-5.4269750 .467616 \quad-1.763942$

H $-6.133860 \quad-0.328021 \quad-0.341849$

H $-7.2971451 .812826 \quad-0.830250$

H $-6.475101 \quad 1.9010290 .737420$

H $-5.7684322 .698179-0.686279$

\section{Partition B HQ:LQ:MM(MULLIKEN)}

48

N $4.675284 \quad-0.136667 \quad-0.769497$

H $4.896508 \quad-1.009259-0.224257$

H $5.014305 \quad-0.239727 \quad-1.729355$

H $\begin{array}{llll}5.110890 & 0.689013 & -0.346669\end{array}$

$\begin{array}{lll}\text { C } 3.151019 & -0.084568 & -0.708918\end{array}$

H $2.7595730 .186796 \quad-1.695515$

C $2.763102 \quad-1.542906-0.309416$

$\begin{array}{llll}0 & 3.633636 & -2.259241 & 0.175303\end{array}$

N $1.487636 \quad-1.883590 \quad-0.591019$

H $0.868131 \quad-1.129316 \quad-0.930976$

C 2.6683620 .9086220 .312478

H 3.1111270 .7164011 .325115

H 1.5539750 .7645600 .417987

C $2.9056962 .346026 \quad-0.110024$

H $3.9937972 .603916 \quad-0.066943$

H $2.5522302 .480787 \quad-1.167962$

C 2.1198273 .2851990 .786190

H 1.0260153 .0285340 .696123

H 2.4088863 .1186201 .856066

C 2.3424134 .7255590 .404378

H 1.7338625 .3880911 .066403

H 3.4173965 .0044000 .508246

H $2.0263144 .898789 \quad-0.653021$

$\begin{array}{llll}\text { C } & 0.818641 & -3.047599 & -0.022709\end{array}$

H $1.502457 \quad-3.5608540 .710117$

H $0.571696 \quad-3.755157 \quad-0.863141$

C $-0.466498 \quad-2.6048870 .706460$

$\begin{array}{llll}0 & -0.554021 & -2.718351 & 1.935502\end{array}$

$\begin{array}{lll}\mathrm{N}-1.508035 & -2.111011 & -0.080630\end{array}$

H $-1.275676 \quad-2.021641 \quad-1.055094$

$\begin{array}{llll}C & -2.085314 & -0.828358 & 0.290847\end{array}$

H $-2.229954 \quad-0.7804741 .373043$

C $-1.0979310 .304159 \quad-0.087408$

$\begin{array}{llll}0 & -0.367133 & 0.128120 & -1.096365\end{array}$

O -1.0164891 .2912490 .671262$

$\begin{array}{llll}\text { C } & -3.442967 & -0.703847 & -0.421644\end{array}$

C - -4.2200170 .5323860 .057906$

$\begin{array}{llll}C & -5.573399 & 0.611117 & -0.656526\end{array}$

$\begin{array}{llll}C & -6.336071 & 1.848178 & -0.168737\end{array}$

H $-4.031693 \quad-1.599742 \quad-0.212365$

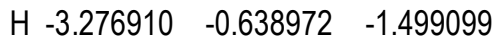

H -4.3768170 .4685191 .137351$

H $-3.6408331 .437878 \quad-0.148353$

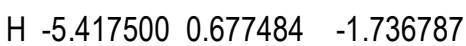

H $-6.157320 \quad-0.288278 \quad-0.442921$

H $-7.3013151 .910509-0.673602$

H -6.5061781 .7858990 .907680$

H $-5.7662952 .753387-0.386933$

Partition B HQ:MM:MM(MULLIKEN)

48

N $4.263306 \quad 0.036323 \quad-1.409656$

H $4.782375 \quad-0.732266 \quad-0.922941$

H $4.338837-0.127172 \quad-2.423136$

H $4.7016490 .946306 \quad-1.218702$

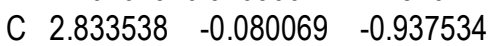

H $2.150465 \quad 0.233530 \quad-1.737424$

$\begin{array}{lll}\text { C } 2.672960 & -1.555976 & -0.521832\end{array}$

$\begin{array}{llll}0 & 3.663899 & -2.148655 & -0.120798\end{array}$

N $1.412184 \quad-2.015639 \quad-0.563021$

H $0.677841 \quad-1.357698 \quad-0.884299$

C 2.5845830 .8098270 .296667

H 3.3626010 .6524541 .047990

H 1.6281130 .5284130 .758557

C $2.5083022 .288786 \quad-0.110584$

H $3.4506442 .615761 \quad-0.551800$

H $1.6971192 .423961 \quad-0.837894$

C 2.1970023 .1375811 .125784

H 1.2172972 .8491291 .532561

H 2.9550602 .9762251 .896398

C 2.1745624 .6192710 .728004

H 1.9525545 .2327571 .603424

H 3.1418374 .9218090 .324271

H $1.398684 \quad 4.796372 \quad-0.020453$

$\begin{array}{llll}\text { C } & 0.894982 & -3.035249 & 0.373217\end{array}$

H $1.634825 \quad-3.2842491 .138198$

H $0.599353 \quad-3.946103 \quad-0.146224$

C $-0.315268 \quad-2.450094 \quad 1.084013$

$\begin{array}{llll}\mathrm{O} & -0.282177 & -2.221398 & 2.285854\end{array}$

N $-1.306764 \quad-2.066717 \quad 0.280273$

$\begin{array}{lll}\mathrm{H}-1.218880 & -2.361528 & -0.678001\end{array}$

$\begin{array}{llll}\text { C } & -1.928854 & -0.744758 & 0.412369\end{array}$

H $-2.079954 \quad-0.5095761 .468841$

$\begin{array}{llll}C & -0.988930 & 0.344870 & -0.157934\end{array}$

$\begin{array}{llll}0 & -0.320118 & 0.059965 & -1.183566\end{array}$

O $-0.88668991 .415683 \quad 0.474108$

$\begin{array}{llll}\text { C } & -3.288443 & -0.794520 & -0.305576\end{array}$

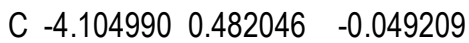

$\begin{array}{llll}C & -5.458988 & 0.390479 & -0.760905\end{array}$

C $-6.2612841 .669520 \quad-0.495883$

H $-3.849212 \quad-1.6568790 .061307$

H -3.121775 $\quad-0.914193 \quad-1.378322$

H -4.2616540 .6059001 .025056$

H $-3.5544861 .354567 \quad-0.415076$

$\begin{array}{llll}H & -5.303574 & 0.270434 & -1.836624\end{array}$

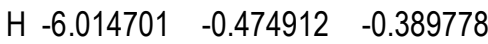

H $-7.2272251 .611450 \quad-0.999973$

H -6.431214 1.7930820 .575262

H $-5.7203362 .539609 \quad-0.872772$ 
Partition B HQ:MM:MM(MULLIKEN) (EE) 48

N $4.2435690 .128073-1.385046$

H $4.769517 \quad-0.627371 \quad-0.883721$

H $4.351013 \quad-0.037713 \quad-2.394808$

H $4.6636271 .045357 \quad-1.187124$

C $2.803034-0.017814 \quad-0.936915$

H $2.1283260 .286394-1.741516$

$\begin{array}{llll}\text { C } & 2.681227 & -1.504454 & -0.540125\end{array}$

$\begin{array}{llll}0 & 3.682701 & -2.073387 & -0.126862\end{array}$

$\begin{array}{llll}\mathrm{N} & 1.442379 & -2.007946 & -0.654300\end{array}$

H $0.684724 \quad-1.357821 \quad-0.922709$

C 2.5298270 .8719230 .295803

H 3.3436850 .7832171 .018904

H 1.6240150 .5283910 .808886

C $2.3378582 .339361 \quad-0.126857$

H $3.2167312 .690208-0.669023$

H $1.4626502 .403112 \quad-0.786081$

C 2.0927823 .2522631 .086022

H 1.1485292 .9866971 .586479

H 2.9064383 .1656261 .812049

C 2.0111944 .7033750 .597230

H 1.8396765 .3705231 .446367

H 2.9397855 .0065230 .110059

H $1.1790484 .832394 \quad-0.099900$

$\begin{array}{llll}\text { C } & 0.926398 & -3.076649 & 0.223078\end{array}$

H $1.671675-3.3703510 .966565$

$\begin{array}{llll}\text { H } & 0.629081 & -3.954235 & -0.348907\end{array}$

C $-0.287636-2.5415430 .985140$

$\begin{array}{llll}\mathrm{O} & -0.184710 & -2.309909 & 2.182933\end{array}$

$\begin{array}{llll}\text { N }-1.372055 & -2.197672 & 0.273033\end{array}$

H - $1.514732 \quad-2.542551 \quad-0.664715$

C - $-1.974464 \quad-0.8702170 .469243$

H -2.219209 $-0.722811 \quad 1.526147$

$\begin{array}{llll}\text { C } & -0.962457 & 0.236632 & 0.084513\end{array}$

$\begin{array}{lllll}0 & -0.254389 & 0.052727 & -0.938531\end{array}$

$\begin{array}{llll}0 & -0.762440 & 1.167545 & 0.885866\end{array}$

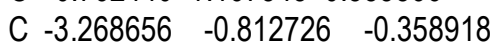

$\begin{array}{lll}C-4.065540 & 0.466131 & -0.055878\end{array}$

$\begin{array}{llll}\text { C } & -5.357260 & 0.482494 & -0.879839\end{array}$

C $-6.1422031 .761549 \quad-0.568049$

H -3.890808 $-1.677339 \quad-0.113369$

$\begin{array}{lll}\text { H }-3.026561 & -0.844111 & -1.423714\end{array}$

H -4.3147280 .5027941 .008028$

H $-3.4701471 .352121 \quad-0.296761$

H $-5.1223620 .450265 \quad-1.947391$

H $-5.970228 \quad-0.388720 \quad-0.631562$

H $-7.0665501 .778662 \quad-1.148816$

H -6.3999631 .8011690 .492210$

H -5.554407 $2.644609-0.825338$

Partition B MM:MM:MM(MULLIKEN)

48

N $4.2684520 .192630 \quad-1.392029$

H $4.932886 \quad-0.217287 \quad-0.744088$
H $4.391431 \quad-0.272787 \quad-2.280490$

H $4.4318141 .186168 \quad-1.470643$

$\begin{array}{lll}\text { C } 2.904866 & -0.062991 & -0.845582\end{array}$

H $2.1158530 .157152 \quad-1.580229$

$\begin{array}{llll}\text { C } 2.693162 & -1.536863 & -0.451575\end{array}$

$\begin{array}{llll}0 & 3.648297 & -2.235642 & -0.133287\end{array}$

N $1.435094 \quad-1.949594 \quad-0.459457$

H $0.773647 \quad-1.207044 \quad-0.714650$

C 2.6765890 .8701500 .355953

H 3.4949080 .7850521 .075408

H 1.7595880 .5619750 .878999

C $2.4945382 .327771 \quad-0.097963$

H $3.3979722 .700711 \quad-0.581613$

H $1.6509782 .388823 \quad-0.798570$

C 2.1731013 .1986661 .120501

H 1.2261732 .8682171 .571228

H 2.9642833 .1099361 .869321

C 2.0483464 .6617580 .675582

H 1.8174725 .2906811 .537760

H 2.9820245 .0079570 .229971

H $1.2393704 .767095 \quad-0.051092$

$\begin{array}{llll}\text { C } & 0.858713 & -3.116653 & 0.238737\end{array}$

H $1.524058 \quad-3.4672341 .031348$

H $0.654788 \quad-3.938107 \quad-0.447054$

C $-0.442351 \quad-2.6749780 .893194$

O $-0.571194 \quad-2.7027112 .109840$

$\begin{array}{lll}\mathrm{N}-1.326506 & -2.119971 & 0.063788\end{array}$

$\begin{array}{lll}\mathrm{H}-1.109420 & -2.226864 & -0.913388\end{array}$

$\begin{array}{lll}\text { C }-1.937848 & -0.818798 & 0.356877\end{array}$

H $-2.152551 \quad-0.7408731 .425724$

C -0.9472800 .3203160 .013388$

$\begin{array}{llll}0 & -0.236467 & 0.185476 & -1.014998\end{array}$

$\begin{array}{llll}0 & -0.845736 & 1.270282 & 0.815248\end{array}$

$\begin{array}{llll}\text { C } & -3.249394 & -0.727978 & -0.441539\end{array}$

$\begin{array}{llll}\text { C } & -4.059790 & 0.517371 & -0.048434\end{array}$

$\begin{array}{llll}\text { C }-5.365782 & 0.567148 & -0.848452\end{array}$

$\begin{array}{lll}\text { C }-6.162599 & 1.813408 & -0.446198\end{array}$

H $-3.845557 \quad-1.620850-0.240963$

H $-3.016878-0.693870 \quad-1.508099$

H $-4.284300 \quad 0.4843161 .020457$

H -3.472580 $1.419917 \quad-0.244759$

H $-5.1427030 .602882 \quad-1.918335$

H $-5.958560 \quad-0.328912 \quad-0.645414$

H $-7.0946191 .855093 \quad-1.012005$

H -6.3998541 .7817810 .618815$

H $-5.5843292 .715404 \quad-0.655109$

Partition C HQ:HQ:HQ

48

N $4.363496 \quad 0.346213 \quad-0.946624$

H $4.759135 \quad-0.439184 \quad-0.347190$

H $4.6652950 .209823 \quad-1.914388$

H $4.6640411 .271141 \quad-0.630384$

C $2.8729110 .120608 \quad-0.745154$

H $2.3339080 .305471 \quad-1.675206$

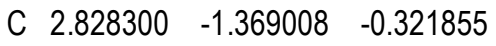

$\begin{array}{llll}0 & 3.859585 & -1.846138 & 0.191114\end{array}$

$\begin{array}{llll}\text { N } & 1.662089 & -1.953774 & -0.552259\end{array}$

H $0.914705 \quad-1.312938 \quad-0.951776$

C 2.3671941 .0586130 .360344

H 3.0424690 .9868391 .226695

H 1.3922300 .6925680 .686352

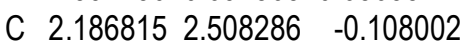

H $3.1373842 .931817-0.483140$

H $1.4762892 .499998 \quad-0.942988$

C 1.6302573 .4082631 .002093

H 0.6607122 .9959391 .303013

H 2.2909253 .3680471 .880695

C 1.4555974 .8616300 .553022

H 1.0439775 .4763081 .361038

H 2.4106905 .3096590 .248105

H $0.7669104 .927336 \quad-0.297236$

C $1.156129 \quad-3.1484910 .133350$

H $1.894171 \quad-3.5178090 .844934$

H $0.922605-3.932542 \quad-0.597546$

C $-0.099032 \quad-2.6414250 .882266$

$\begin{array}{llll}\mathrm{O} & -0.096480 & -2.484326 & 2.092870\end{array}$

N $-1.118341 \quad-2.2517740 .042908$

H $-0.906195 \quad-2.356853 \quad-0.944862$

C $-1.782303 \quad-0.949947 \quad 0.268029$

H $-1.819799 \quad-0.7949701 .348043$

$\begin{array}{llll}C & -0.904513 & 0.188261 & -0.364568\end{array}$

$\begin{array}{llll}0 & -0.134170 & -0.166721 & -1.333718\end{array}$

O -1.0104831 .3263440 .116755$

$\begin{array}{llll}\text { C } & -3.207935 & -0.966639 & -0.309843\end{array}$

C -4.0459800 .2609210 .070837$

$\begin{array}{llll}C & -5.440656 & 0.253630 & -0.567555\end{array}$

C $-6.287254 \quad 1.467345 \quad-0.170219$

H $-3.705027-1.8811570 .041060$

H -3.145135 $-1.044817 \quad-1.406052$

H -4.1471260 .2990951 .165486$

H $-3.5070101 .172017 \quad-0.212235$

$\begin{array}{llll}H & -5.338351 & 0.222761 & -1.662083\end{array}$

H $-5.969676 \quad-0.668852 \quad-0.286364$

$\begin{array}{llll}H & -7.276359 & 1.440698 & -0.642736\end{array}$

H -6.4372731 .5060420 .915884$

H $-5.7989872 .403360 \quad-0.467945$

\section{Partition C HQ:HQ:LQ}

48

$\begin{array}{llll}\text { N } & 4.643880 & 0.149482 & -0.121650\end{array}$

H $4.754482 \quad-0.7871360 .375978$

H $\begin{array}{llll}5.289615 & 0.212267 & -0.908814\end{array}$

H 4.8257050 .9277900 .517330

$\begin{array}{llll}\text { C } 3.171443 & 0.070017 & -0.552279\end{array}$

H $3.1133520 .269896 \quad-1.625841$

C $\begin{array}{lll}2.816852 & -1.414023 & -0.210382\end{array}$

$\begin{array}{llll}0 & 3.651414 & -2.057464 & 0.444703\end{array}$

N $1.657532 \quad-1.864244 \quad-0.703547$

H $1.009652 \quad-1.190813 \quad-1.158311$

C 2.3198371 .0413340 .206127

H 2.3565950 .8415641 .309924 
H $1.2340090 .884160 \quad-0.115249$

$\begin{array}{lll}\text { C } 2.656668 & 2.490393 & -0.075964\end{array}$

H 3.6809782 .7510970 .300918

H $2.6481212 .672614 \quad-1.183175$

C 1.6298923 .3885180 .588646

H 0.5930793 .0292170 .322697

H 1.7270623 .3000261 .702721

C 1.8077534 .8243560 .172627

H 1.0563135 .4672890 .691957

H 2.8290395 .1940930 .433311

H $1.6633064 .937639-0.929188$

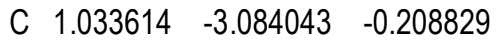

H $1.711095-3.6034840 .524997$

H $0.848075 \quad-3.764914 \quad-1.085224$

$\begin{array}{llll}\text { C } & -0.300530 & -2.746039 & 0.499835\end{array}$

$\begin{array}{llll}\mathrm{O} & -0.400799 & -2.963137 & 1.716951\end{array}$

$\mathrm{N}-1.338278 \quad-2.248447 \quad-0.290789$

H $-1.112829 \quad-2.209209-1.268477$

C - $-1.953451 \quad-0.9895900 .134223$

H -2.054391 -1.0115581 .258173$

C $-1.0521150 .217878 \quad-0.253004$

$\begin{array}{llll}0 & -0.310229 & 0.123063 & -1.294182\end{array}$

O -1.0181381 .2333610 .485030$

$\begin{array}{llll}\text { C } & -3.340488 & -0.801708 & -0.489772\end{array}$

C -4.0925020 .2957820 .233558$

C $-5.3900760 .624439 \quad-0.473274$

C -6.1603071 .6931010 .258553$

H -3.926334 $-1.756216 \quad-0.432676$

$\begin{array}{lll}\text { H }-3.225421 & -0.536564 & -1.573406\end{array}$

H $-4.309081 \quad-0.0278641 .285641$

H -3.444282 1.2124550 .296262

H -5.169061 $0.969274 \quad-1.518057$

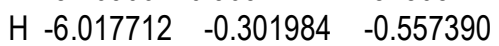

H -7.107932 $1.930236-0.282413$

H -6.4183791 .3553961 .291409$

H -5.5545322 .6281210 .338402$

\section{Partition C HQ:LQ:LQ}

48

N $3.787554 \quad 0.346320 \quad-1.543530$

H $4.6031180 .057235 \quad-0.982640$

H $3.774268 \quad-0.181917 \quad-2.423279$

H $3.8478951 .353430 \quad-1.743779$

$\begin{array}{llll}\text { C } 2.532892 & 0.071957 & -0.766134\end{array}$

H $1.6242430 .240998 \quad-1.455403$

$\begin{array}{llll}\text { C } & 2.600527 & -1.384475 & -0.231468\end{array}$

$\begin{array}{lllll}0 & 3.580073 & -1.705776 & 0.469940\end{array}$

N $1.617032 \quad-2.251887 \quad-0.651701$

H $0.932451 \quad-1.848606 \quad-1.281869$

C 2.3821691 .0102350 .429174

H 3.1399330 .7708091 .218862

H 1.3485010 .8009150 .848684

C 2.4488962 .4713010 .037570

H $3.5165812 .804278-0.059111$

H $1.9416842 .626438 \quad-0.952547$

C 1.7416593 .3250311 .072014
H 0.6550623 .0273831 .093924

H 2.1711903 .1170292 .086807

C 1.8687364 .7913340 .753406

H 1.3339105 .3977391 .523982

H 2.9400615 .1070510 .738393

H $1.4202785 .018163 \quad-0.244128$

$\begin{array}{llll}\text { C } & 1.069150 & -3.234160 & 0.253910\end{array}$

H $1.819538 \quad-3.4935081 .052344$

H $0.827098 \quad-4.160544 \quad-0.337627$

$\begin{array}{llll}C & -0.214097 & -2.699711 & 0.938315\end{array}$

$\begin{array}{llll}\mathrm{O} & -0.252681 & -2.628410 & 2.175640\end{array}$

$\mathrm{N}-1.287346 \quad-2.3435560 .113047$

H $-1.145309 \quad-2.570919 \quad-0.853377$

$\begin{array}{llll}C & -1.804860 & -0.982227 & 0.244522\end{array}$

H $-1.843061 \quad-0.7303121 .344469$

$\begin{array}{llll}\text { C } & -0.866408 & 0.041145 & -0.456527\end{array}$

$\begin{array}{llll}0 & -0.270601 & -0.301121 & -1.536739\end{array}$

$\begin{array}{llll}0 & -0.663780 & 1.160286 & 0.080759\end{array}$

$\begin{array}{llll}\text { C }-3.212613 & -0.852658 & -0.347797\end{array}$

C -3.8545560 .4387780 .114084$

C $-5.1691190 .678969 \quad-0.596954$

C $-5.8304611 .945858 \quad-0.119007$

H $-3.849188 \quad-1.720246 \quad-0.032452$

$\begin{array}{lll}\text { H }-3.144725 & -0.867581 & -1.467208\end{array}$

H -4.028472 $0.396643 \quad 1.221608$

H -3.150880 $1.294240 \quad-0.077507$

H $-4.9893050 .742812-1.702803$

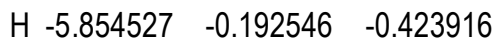

H $-6.7927402 .111442 \quad-0.660748$

H -6.0466521 .8888150 .975282$

H -5.167222 $2.826536 \quad-0.298124$

\section{Partition C LQ:LQ:LQ}

48

$\begin{array}{llll}\text { N } 3.781944 & 0.345829 & -1.548511\end{array}$

H $4.5949870 .063856 \quad-0.989216$

H $3.767251 \quad-0.185373-2.419680$

H $3.8357721 .347052 \quad-1.752878$

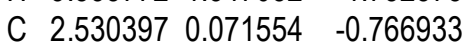

H $1.6190410 .240388 \quad-1.453172$

$\begin{array}{llll}\text { C } & 2.599234 & -1.384745 & -0.231834\end{array}$

$\begin{array}{lllll}0 & 3.579281 & -1.705232 & 0.469148\end{array}$

$\begin{array}{llll}\mathrm{N} & 1.615892 & -2.252807 & -0.651159\end{array}$

$\begin{array}{llll}\text { H } & 0.931159 & -1.850482 & -1.281710\end{array}$

C 2.3832721 .0100770 .428724

H 3.1439800 .7713761 .215791

H 1.3512360 .8002270 .851858

C 2.4474962 .4710090 .036372

H $3.5145012 .804275 \quad-0.066331$

H $1.9346212 .625543 \quad-0.950925$

C 1.7456633 .3247251 .074538

H 0.6591453 .0273761 .101703

H 2.1801223 .1164202 .087170

C 1.8715084 .7910820 .755618

H 1.3407595 .3974721 .529020

H 2.9428325 .1065030 .735260
H $1.4180835 .018228 \quad-0.239598$

$\begin{array}{llll}C & 1.068518 & -3.234382 & 0.255462\end{array}$

H $1.819352-3.4930191 .053708$

H $0.826255 \quad-4.161279 \quad-0.335206$

C $-0.214453 \quad-2.6995490 .940108$

$\begin{array}{llll}0 & -0.252525 & -2.627506 & 2.177394\end{array}$

N $-1.288114 \quad-2.3437990 .115120$

H $-1.146864 \quad-2.572493 \quad-0.851112$

$\begin{array}{llll}C & -1.804592 & -0.981986 & 0.245232\end{array}$

H $-1.841804 \quad-0.7286111 .344880$

C $-0.8659320 .039590 \quad-0.457990$

$\begin{array}{llll}0 & -0.272721 & -0.304110 & -1.539246\end{array}$

$\begin{array}{llll}0 & -0.659921 & 1.158528 & 0.078439\end{array}$

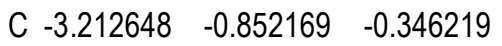

C -3.8530140 .4405800 .114189$

C $-5.1678650 .681015 \quad-0.596282$

$\begin{array}{llll}C & -5.827779 & 1.949136 & -0.119555\end{array}$

H -3.849718 $-1.718725 \quad-0.029064$

$\begin{array}{lll}\text { H }-3.145562 & -0.868843 & -1.465652\end{array}$

H -4.0261650 .4002831 .221904$

H $-3.1486301 .295046 \quad-0.079198$

H $-4.9887440 .743233 \quad-1.702339$

H $-5.853927 \quad-0.189667 \quad-0.421635$

H $-6.7902692 .114868 \quad-0.660881$

H -6.0433101 .8937280 .974950$

H $-5.1638862 .828999 \quad-0.300260$

Partition C HQ:HQ:MM(MULLIKEN)

48

$\begin{array}{llll}\mathrm{N} & 4.263306 & 0.036323 & -1.409656\end{array}$

H $4.782375 \quad-0.732266 \quad-0.922941$

H $4.338837 \quad-0.127172 \quad-2.423136$

H $4.7016490 .946306 \quad-1.218702$

$\begin{array}{lll}\text { C } 2.833538 & -0.080069 & -0.937534\end{array}$

H $2.150465 \quad 0.233530 \quad-1.737424$

C $2.672960-1.555976 \quad-0.521832$

$\begin{array}{llll}0 & 3.663899 & -2.148655 & -0.120798\end{array}$

$\begin{array}{llll}\text { N } & 1.412184 & -2.015639 & -0.563020\end{array}$

H $0.6778411-1.357698 \quad-0.884299$

C 2.5845830 .8098270 .296667

H 3.3626010 .6524541 .047990

H 1.6281130 .5284130 .758557

C $2.508302 \quad 2.288786 \quad-0.110584$

H $3.4506442 .615761 \quad-0.551800$

H $1.6971192 .423961 \quad-0.837894$

C 2.1970023 .1375811 .125784

H 1.2172972 .8491291 .532561

H 2.9550602 .9762251 .896398

C 2.1745624 .6192710 .728004

H 1.9525545 .2327571 .603424

H 3.1418374 .9218090 .324271

H $1.398684 \quad 4.796372 \quad-0.020453$

$\begin{array}{llll}\text { C } & 0.894982 & -3.035249 & 0.373217\end{array}$

H $1.634825 \quad-3.2842491 .138198$

H $0.599353 \quad-3.946103 \quad-0.146224$

$\begin{array}{llll}C & -0.315268 & -2.450094 & 1.084013\end{array}$ 


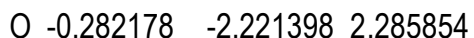

N $-1.306764 \quad-2.066717 \quad 0.280273$

H $-1.218880 \quad-2.361528 \quad-0.678001$

C - $-1.928854 \quad-0.7447580 .412369$

H $-2.079954 \quad-0.5095761 .468841$

C $-0.9889300 .344870 \quad-0.157933$

$\begin{array}{llll}0 & -0.320118 & 0.059965 & -1.183566\end{array}$

O -0.8868991 .4156830 .474108$

C $-3.288443-0.794520 \quad-0.305576$

$\begin{array}{llll}\text { C } & -4.104990 & 0.482046 & -0.049209\end{array}$

C $-5.4589880 .390479 \quad-0.760905$

C $-6.2612841 .669520 \quad-0.495883$

H $-3.849212 \quad-1.6568790 .061307$

H $-3.121775 \quad-0.914193 \quad-1.378323$

H -4.2616540 .6059001 .025056$

H $-3.5544861 .354567 \quad-0.415076$

H $-5.3035740 .270434 \quad-1.836624$

H $-6.014701 \quad-0.474912 \quad-0.389779$

H $-7.2272251 .611450 \quad-0.999973$

H -6.4312151 .7930820 .575262$

H $-5.7203362 .539609-0.872772$

Partition C HQ:HQ:MM(MULLIKEN) (EE) 48

N $4.2435690 .128073-1.385046$

H $4.769517-0.627371 \quad-0.883721$

H $4.351013 \quad-0.037713 \quad-2.394808$

H $4.6636271 .045357-1.187124$

$\begin{array}{lll}\text { C } 2.803034 & -0.017814 & -0.936915\end{array}$

H $2.1283260 .286394 \quad-1.741516$

C $2.681227-1.504454-0.540125$

$\begin{array}{llll}\text { O } & 3.682701 & -2.073387 & -0.126862\end{array}$

N $1.442379-2.007946 \quad-0.654300$

H $0.684724-1.357821 \quad-0.922709$

C 2.5298270 .8719230 .295803

H $3.3436850 .783217 \quad 1.018904$

H 1.6240150 .5283910 .808886

C $2.3378582 .339361-0.126857$

H $3.2167312 .690208-0.669023$

H $1.4626502 .403112 \quad-0.786081$

C 2.0927823 .2522631 .086022

H 1.1485292 .9866971 .586479

H 2.9064383 .1656261 .812049

C 2.0111944 .7033750 .597230

H $1.839676 \quad 5.3705231 .446367$

H 2.9397855 .0065230 .110059

H $1.1790484 .832394-0.099900$

C $0.926398 \quad-3.0766490 .223078$

H $1.671675-3.3703510 .966565$

H $0.629081 \quad-3.954235 \quad-0.348907$

C $-0.287636 \quad-2.5415430 .985140$

O $-0.184710 \quad-2.3099092 .182933$

N $-1.372055-2.1976720 .273033$

H -1.514732 $-2.542551 \quad-0.664715$

C $-1.974464 \quad-0.8702170 .469243$

H $-2.219209-0.7228111 .526147$
C $-0.962457 \quad 0.236632 \quad 0.084513$

$\begin{array}{llll}0 & -0.254389 & 0.052727 & -0.938531\end{array}$

$\begin{array}{lllll}0 & -0.762440 & 1.167545 & 0.885866\end{array}$

$\begin{array}{lll}\text { C }-3.268656 & -0.812726 & -0.358918\end{array}$

$\begin{array}{lll}\text { C }-4.065540 & 0.466131 & -0.055878\end{array}$

C $-5.3572600 .482494 \quad-0.879839$

C $-6.1422031 .761549 \quad-0.568049$

H $-3.890808 \quad-1.677339-0.113369$

H $-3.026561 \quad-0.844111 \quad-1.423714$

H $-4.3147280 .502794 \quad 1.008028$

H $-3.4701471 .352121 \quad-0.296761$

H $-5.1223620 .450265 \quad-1.947391$

H $-5.970228 \quad-0.388720 \quad-0.631562$

H $-7.0665501 .778662 \quad-1.148816$

H -6.3999631 .8011690 .492210$

H $-5.5544072 .644609 \quad-0.825338$

Partition C HQ:MM:MM(MULLIKEN)

48

N $4.2837330 .193204-1.392934$

H $4.962025 \quad-0.228837 \quad-0.735868$

H $4.398617 \quad-0.270469-2.304849$

H $4.4497991 .205458-1.467423$

$\begin{array}{lll}\text { C } 2.904926 & -0.063521 & -0.842894\end{array}$

H $2.1175800 .157380 \quad-1.578719$

C $2.692648-1.537096-0.449682$

$\begin{array}{llll}0 & 3.647297 & -2.236457 & -0.130930\end{array}$

N $1.434415 \quad-1.949474 \quad-0.458840$

H $0.773638-1.206420 \quad-0.714232$

C 2.6758940 .8695420 .358471

H $3.4945730 .784623 \quad 1.077397$

H 1.7588980 .5612890 .881367

C $2.4940192 .326732 \quad-0.096552$

H $3.3975122 .699064-0.580430$

H $1.6505652 .387113 \quad-0.797329$

C 2.1724143 .1986081 .121150

H 1.2254082 .8684211 .571905

H 2.9634403 .1103841 .870187

C 2.0477164 .6613690 .675116

H 1.8166145 .2909071 .536781

H 2.9814655 .0072570 .229434

H $1.2388714 .766078 \quad-0.051798$

C $0.857161 \quad-3.1168680 .238099$

H $1.521845 \quad-3.4680901 .030973$

H $0.653694 \quad-3.937786 \quad-0.448457$

$\begin{array}{llll}\text { C }-0.444427 & -2.675619 & 0.891838\end{array}$

$\begin{array}{llll}0 & -0.574332 & -2.704380 & 2.108352\end{array}$

$\mathrm{N}-1.327746 \quad-2.1196730 .062181$

H -1.109465 $-2.225412 \quad-0.914858$

$\begin{array}{lll}\text { C - }-1.939101 & -0.818671 & 0.355938\end{array}$

H $-2.153975 \quad-0.7413241 .424789$

C -0.9483290 .3205230 .013320$

O

O -0.8472831 .2702960 .815486$

$\begin{array}{lll}\text { C }-3.250476 & -0.727303 & -0.442686\end{array}$

C $-4.0609550 .517810 \quad-0.049019$
C $-5.366780 \quad 0.568109 \quad-0.849278$

C $-6.1636991 .814101 \quad-0.446397$

H $-3.846686 \quad-1.620294 \quad-0.242792$

H $-3.017712 \quad-0.692556 \quad-1.509169$

H -4.285676 $0.484107 \quad 1.019805$

H -3.473675 $1.420456-0.244676$

H $-5.1434560 .604537-1.919086$

H $-5.959587 \quad-0.328090 \quad-0.646947$

H -7.095581 $1.856144 \quad-1.012402$

H $-6.401190 \quad 1.7817680 .618542$

H $-5.5853682 .716226-0.654576$

Partition C HQ:MM:MM (MULLIKEN) (EE)

48

N - 1.6110010 .3361371 .955219

H -2.148592 -0.0425252 .742051$

H -0.5773780 .1872502 .074241$

H $-1.761417 \quad 1.3484701 .856326$

$\begin{array}{lll}\text { C }-2.015058 & -0.333767 & 0.664007\end{array}$

H $-1.304848 \quad-0.055357 \quad-0.128737$

$\begin{array}{llll}\text { C }-1.951814 & -1.896718 & 0.664864\end{array}$

$\begin{array}{llll}0 & -2.977325 & -2.551195 & 0.520439\end{array}$

N $-0.754973 \quad-2.5015500 .675774$

H $0.011740 \quad-1.9727921 .105207$

C -3.392306 0.2098690 .228538

H -4.1182020 .0416111 .027627$

H $-3.731242 \quad-0.384837 \quad-0.628776$

C $-3.3746361 .699794 \quad-0.178212$

H -3.0397872 .3255650 .651256$

H $-2.7008841 .866021-1.031277$

C $-4.7904132 .135637 \quad-0.569841$

H $-5.1546521 .559103 \quad-1.429536$

H -5.4813881 .9792430 .263959$

C $-4.768363 \quad 3.627423 \quad-0.927418$

H $-5.7732513 .959420-1.200655$

H $-4.4343724 .224106 \quad-0.076189$

H $-4.1065293 .816797-1.776109$

C $-0.265273 \quad-3.094891 \quad-0.586208$

H $-1.084021 \quad-3.485479-1.198926$

H $0.457665 \quad-3.893345 \quad-0.410496$

$\begin{array}{llll}\text { C } & 0.391036 & -1.948350 & -1.335588\end{array}$

$\begin{array}{llll}\mathrm{O} & -0.307373 & -1.168983 & -1.974569\end{array}$

N $1.639338 \quad-1.671074 \quad-0.962375$

H $2.140557 \quad-2.380112 \quad-0.451137$

$\begin{array}{llll}\text { C } 2.049266 & -0.291237 & -0.669014\end{array}$

H $1.8908370 .332294 \quad-1.553412$

C $1.164590 \quad 0.2862920 .458575$

$\begin{array}{llll}\text { O } & 0.943513 & -0.415963 & 1.476986\end{array}$

O 0.5050491 .3242850 .251863

$\begin{array}{lll}\text { C } 3.546189 & -0.303475 & -0.319875\end{array}$

C $4.1011821 .123059-0.180648$

C 5.5993041 .0683540 .135433

C 6.1434582 .4946140 .274393

H $4.092566 \quad-0.818065 \quad-1.114548$

H $3.698193 \quad-0.8474610 .615573$

H $3.9483731 .670954-1.114163$ 
H 3.5817001 .6556870 .620841

H 5.7659790 .5211011 .067635

H $6.1317120 .552827 \quad-0.669117$ H 7.2122832 .4612350 .494534 H $5.9992923 .048932-0.655056$ H 5.6382463 .0199781 .086772

Partition C LQ:LQ:MM (MULLIKEN)
48

N $3.9755480 .126673 \quad-1.703086$

H $4.753718-0.375052 \quad-1.245372$

H $3.886862 \quad-0.223729-2.666778$

H $4.189345 \quad 1.130353 \quad-1.748019$

$\begin{array}{llll}\text { C } & 2.700392 & -0.102141 & -0.973360\end{array}$

H $1.8432480 .170755 \quad-1.667806$

$\begin{array}{lll}\text { C } 2.604131 & -1.566017 & -0.486827\end{array}$

$\begin{array}{lllll}0 & 3.614086 & -2.076646 & 0.025680\end{array}$

$\begin{array}{llll}\mathrm{N} & 1.396000 & -2.190436 & -0.607129\end{array}$

H $0.652858 \quad-1.617941 \quad-1.015335$

$\begin{array}{llll}\text { C } 2.584677 & 0.797276 & 0.309687\end{array}$

H 3.4448680 .6394130 .965605

H 1.6864670 .4999120 .870078

C $2.4572112 .279894 \quad-0.073941$

H $3.3618022 .626530 \quad-0.574597$

H $1.5945332 .412063 \quad-0.740232$

C 2.2244743 .1100921 .191648

H 1.2803762 .8034281 .662385

H 3.0382802 .9499851 .903652

C 2.1538964 .5956560 .814508

H 1.9859965 .1965511 .710425

H 3.0864454 .9162750 .347842

H 1.3254164 .7695670 .123744

$\begin{array}{llll}\text { C } & 0.906728 & -3.065408 & 0.462976\end{array}$

H $1.690754-3.2808691 .194065$

H $0.521121 \quad-4.0054070 .070010$

C $-0.206337 \quad-2.3320041 .193597$

$\begin{array}{llll}0 & -0.030880 & -1.901099 & 2.325921\end{array}$

$\begin{array}{lll}\mathrm{N}-1.276802 & -2.054332 & 0.450734\end{array}$

$\begin{array}{lll}\mathrm{H}-1.302986 & -2.500089 & -0.450910\end{array}$

$\begin{array}{lll}\text { C }-1.865111 & -0.711054 & 0.442970\end{array}$

H $-1.939805 \quad-0.3338271 .465781$

C $-0.9464480 .267338 \quad-0.327462$

$\begin{array}{llll}0 & -0.356957 & -0.166369 & -1.349531\end{array}$

O -0.7847741 .4094570 .147941$

$\begin{array}{llll}C & -3.271195 & -0.819580 & -0.171542\end{array}$

$\begin{array}{lll}\text { C }-4.045237 & 0.501324 & -0.036927\end{array}$

$\begin{array}{lll}\text { C }-5.446029 & 0.352273 & -0.640036\end{array}$

$\begin{array}{llll}\text { C } & -6.205189 & 1.676461 & -0.498401\end{array}$

H -3.821340 -1.6098480 .343779$

H -3.180216 $-1.085205 \quad-1.227061$

H -4.126140 0.7715631 .018690

H -3.505463 $1.302026-0.552376$

H $-5.3666070 .085606 \quad-1.697559$

H $-5.990802 \quad-0.440479 \quad-0.120192$

H $-7.2041431 .577665 \quad-0.926067$

H -6.299619 1.9464690 .555005
H $-5.6757702 .473543 \quad-1.023839$

Partition C LQ:MM:MM (MULLIKEN) 48

N $4.281454 \quad 0.193432 \quad-1.394118$

H $4.957829-0.226670-0.740153$

H $4.393476 \quad-0.265661 \quad-2.302158$

H $4.441696 \quad 1.200168 \quad-1.468623$

$\begin{array}{llll}\text { C } 2.904100 & -0.063679 & -0.843316\end{array}$

H $2.1158530 .157042 \quad-1.578324$

$\begin{array}{llll}\text { C } & 2.692355 & -1.537297 & -0.449842\end{array}$

$\begin{array}{llll}0 & 3.647194 & -2.236057 & -0.130515\end{array}$

$\begin{array}{llll}\text { N } & 1.434317 & -1.950236 & -0.459422\end{array}$

H $0.773319-1.207528 \quad-0.715271$

C 2.6759290 .8693020 .358231

H 3.4950840 .7843821 .076648

H 1.7593060 .5609670 .881772

C $2.4936592 .326526 \quad-0.096570$

H $3.3967152 .698941 \quad-0.581227$

H $1.6495082 .387019 \quad-0.796517$

C 2.1732193 .1983091 .121505

H 1.2266292 .8681251 .573127

H 2.9649653 .1100501 .869782

C 2.0481214 .6610950 .675658

H 1.8178685 .2906001 .537575

H 2.9814505 .0069930 .229100

H $1.2385794 .765869 \quad-0.050471$

$\begin{array}{llll}\text { C } & 0.857217 & -3.117095 & 0.238530\end{array}$

H $1.522204 \quad-3.4679831 .031305$

H $0.653207 \quad-3.938386-0.447420$

C $-0.443934-2.6750670 .892621$

$\begin{array}{llll}0 & -0.573136 & -2.702686 & 2.109236\end{array}$

N -1.327628 -2.1196730 .062986$

H $-1.110077-2.226404-0.914106$

C $-1.938621 \quad-0.8183310 .356018$

H $-2.153225 \quad-0.7402711 .424873$

C -0.9476990 .3204710 .012469$

$\begin{array}{llll}0 & -0.236668 & 0.185197 & -1.015700\end{array}$

$\begin{array}{llll}0 & -0.846384 & 1.270772 & 0.813974\end{array}$

$\begin{array}{lll}\text { C }-3.250192 & -0.727253 & -0.442331\end{array}$

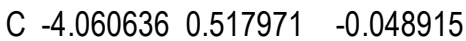

C $-5.3667770 .567754 \quad-0.848693$

$\begin{array}{llll}\text { C } & -6.163665 & 1.813844 & -0.446047\end{array}$

$\begin{array}{lll}\mathrm{H}-3.846334 & -1.620177 & -0.241930\end{array}$

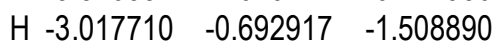

H -4.284956 0.4847201 .020008

H $-3.4735611 .420610 \quad-0.245212$

H $-5.1438790 .603735 \quad-1.918605$

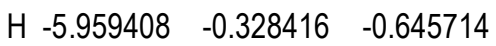

H $-7.0957791 .855535 \quad-1.011698$

H -6.400733 1.7819600 .619000

H $-5.5855182 .715939 \quad-0.654862$

Partition C MM:MM:MM (MULLIKEN) 48

N $4.2684520 .192630 \quad-1.392029$ $\begin{array}{llll}\text { H } & 4.932886 & -0.217287 & -0.744088\end{array}$

H $\begin{array}{lll}4.391431 & -0.272787 & -2.280490\end{array}$

H $4.431814 \quad 1.186168 \quad-1.470643$

$\begin{array}{llll}\text { C } & 2.904866 & -0.062991 & -0.845582\end{array}$

H $2.1158530 .157152 \quad-1.580229$

$\begin{array}{lll}\text { C } 2.693162 & -1.536863 & -0.451575\end{array}$

$\begin{array}{llll}0 & 3.648297 & -2.235642 & -0.133287\end{array}$

$\begin{array}{llll}\mathrm{N} & 1.435094 & -1.949594 & -0.459457\end{array}$

H $0.773647 \quad-1.207044 \quad-0.714650$

C 2.6765890 .8701500 .355953

H 3.4949080 .7850521 .075408

H 1.7595880 .5619750 .878999

$\begin{array}{llll}\text { C } 2.494538 & 2.327771 & -0.097963\end{array}$

H $3.3979722 .700711 \quad-0.581613$

H $1.6509782 .388823 \quad-0.798570$

C 2.1731013 .1986661 .120501

H 1.2261732 .8682171 .571228

H 2.9642833 .1099361 .869321

C 2.0483464 .6617580 .675582

H 1.8174725 .2906811 .537760

H 2.9820245 .0079570 .229971

H $1.2393704 .767095 \quad-0.051092$

$\begin{array}{llll}\text { C } & 0.858713 & -3.116653 & 0.238737\end{array}$

H $1.524058 \quad-3.4672341 .031348$

$\begin{array}{llll}\text { H } & 0.654788 & -3.938107 & -0.447054\end{array}$

$\begin{array}{llll}C & -0.442351 & -2.674978 & 0.893194\end{array}$

$\begin{array}{llll}0 & -0.571194 & -2.702710 & 2.109840\end{array}$

N -1.326506 -2.1199710 .063789$

H -1.109420 $-2.226864 \quad-0.913388$

C $-1.937848 \quad-0.8187980 .356877$

H $-2.152551 \quad-0.7408731 .425724$

C -0.9472800 .3203160 .013388$

$\begin{array}{llll}0 & -0.236467 & 0.185476 & -1.014998\end{array}$

O

$\begin{array}{lll}\text { C }-3.249394 & -0.727978 & -0.441539\end{array}$

$\begin{array}{llll}C & -4.059790 & 0.517371 & -0.048434\end{array}$

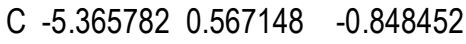

$\begin{array}{llll}C & -6.162599 & 1.813408 & -0.446198\end{array}$

H $-3.845557 \quad-1.620850 \quad-0.240963$

H $-3.016878 \quad-0.693870 \quad-1.508099$

H -4.2843000 .4843161 .020457$

H $-3.472580 \quad 1.419917 \quad-0.244759$

H $-5.1427030 .602882 \quad-1.918335$

H $-5.958560 \quad-0.328912 \quad-0.645414$

H $-7.0946191 .855093 \quad-1.012005$

H -6.399854 1.7817810 .618815

H $-5.5843292 .715404 \quad-0.655109$ 